\title{
Effect of Drying Methods and Pre-Treatments on Nutritional Value and Sensory Quality of Fish (Oreochromis niloticus, Pylodictis olivaris and Cyprinus carpio) Species Commonly Used in Gambella Region
}

\author{
Dagne Tarle $^{1}$, Mitiku Guya ${ }^{2}$, Solomon Abera ${ }^{3}$, Getachew Bekele Fereja ${ }^{1^{*}}$ \\ ${ }^{1}$ Department of Animal science, Collage of Agriculture and Natural Resources, Gambella University, Gambella, Ethiopia \\ ${ }^{2}$ School of Animal Science, College of Agriculture, Haramaya University, Ethiopia \\ ${ }^{3}$ Department of Food Science and Technology, School of Food Science, Postharvest Technology and Process Engineering, Haramaya \\ University, Ethiopia \\ A R T I C L E I N F O \\ Article history: \\ Received 01 August 2015 \\ Accepted 10 December 2015 \\ Available online, ISSN: 2148-127X \\ Keywords \\ Fish \\ Fish fillet \\ Drying \\ Chemical \\ Sensory quality \\ ${ }^{*}$ Corresponding Author: \\ E-mail: gechobek@gmail.com \begin{abstract}
A B S T R A C T
This study was conducted to assess the effect of drying methods and pre-treatments on nutritional content and sensory quality of dried fish. The experiment was conducted in factorial arrangement of $2 \times 3 \times 2$ with two drying methods (sun and oven drying), three fish species (tilapia, cat fish and carp) and two preservatives treatment (garlic and ginger juice) laid out in Completely Randomized Design (CRD). Fresh fillets were analysed for their nutritional value and sensory quality. The compositions of the fresh fillets were 6.50-7.59\% for ash, $74.20-76.67 \%$ for protein, $8.06-9.09 \%$ for fat and $8.47-9.12 \%$ for total carbohydrates. Drying reduced the moisture contents from $74.74-75.81 \%$ to between 7.76-8.25\%, making it safe for storage. The ash content changed from 7.11 to 7.34 and from $6.50 \%$ to $6.34 \%$ for cat fish and tilapia, respectively, with statistical significance whereas no change was observed in carp with $7.60 \%$ because of drying. Drying method had no difference in ash and protein contents while increase in fat from 7.75 to $9.44 \%$ and a decrease in carbohydrate from 9.37 to $8.13 \%$ were observed in sun dried samples than that of oven dried fillets. This study showed that nutritional values of dry fish did not statistically changed during storage period of 3 months.
\end{abstract}

\section{Introduction}

Different species of fish had benefits for the world as food for human consumption (Mdegela et al., 2010). The global traditional and improved fish processing technologies in aquatic food production had more than double since 1970, with a total of approximately 93.2 million metric tons in 1997 (Akinneye et al., 2007 and 2010). Fish was a major source of protein and its harvesting, handling, processing and distribution provide livelihood for people in many countries (Delgade et al, 2003). The use of appropriate technology which is a radical approach to stem up production and processing technique, has became subordinate to social need, and is of paramount importance (Opara and Al Jufaili, 2006). Most commonly, benefits are computed as commodity output or number of fish produced. Commodity output may be further split between the animals harvested by capture (fishing for wild animals) or culture (produced as captive animals) commonly called the capture fisheries and the culture fisheries, respectively (Opara and $\mathrm{Al}$ Jufaili, 2006).

Mostly fish was caught for home consumption in different parts of Ethiopia. According to MoA 2012/13 fiscal year, Ethiopian consumption on fish was mainly depend on Koroso and Ambaza because of their access abundant relative to other spp. For instance, Koroso accounted for $75 \%$ of the fish production followed by Ambaza, with $12 \%$ and other types of fish account for $13 \%$ in which Duba was included was reported.

In history, riverside fishing activities were performed on two main rivers, Baro River which is found near Gambella in the western part of the country and Omo River which is found in the southern area near the border with Kenya (UNCEF, 2005). Alwero reservoir is known by its natural wild fish production in Gambella regional state which is located in Anywaa zone, Abobo District that connected with Baro-River after it passes the zone and goes to Sudane. Eyo (1997) reported that fish was abundantly caught in dry season. Thus, it was imperative to process and preserve some of the fish harvested in the period of abundance, so as to ensure an all year round supply.

The common methods of fish preservation in Ethiopia include drying, salting, smoking, and their combinations (Okorely and Kwarten, 2006). Sun drying was one of the traditional methods employed to preserve fish in Gambella region. It had been observed as the most convenient and cheapest form of preservation (Eyo, 1986). The traditional fish processing activities in Gambella include exposing the whole fish to the open sun drying without slicing; splitting, filleting and sticking after eviscerated the internal organs. The quality of the 
sun-dried fish product was judged on degree of drying, appearance, damaged and insect infestation that influence nutrition values (Bolaji, 2005) under the principles of HACCP. Hence the objective of this study was to study the effects of drying methods and pre-treatments on nutritional values and sensory quality of three fish species.

\section{Materials and Methods}

\section{Experimental Location}

Three experimental fish species namely tilapia (Oreochromis niloticus), flathead cat fish (Pylodictis olivaris) and carp (Cyprinus carpio) were collected from Alwero reservoir, Abobo District in Gambella regional state where drying and pre-treatments were conducted. The analysis for the chemical composition of fresh and dried fish samples such as moisture content, fat, fiber and ash were conducted in the laboratory of Animal Nutrition of Haramaya University.

\section{Experimental Materials}

Fish (three fish species namely, tilapia (Oreochromis niloticus), cat fish (Pylodictis olivaris), and common carp (Cyprinus carpio), Ginger (Zingiber officinale), and Garlic (Allium sativum).

\section{Experimental Design and Treatment Planning}

The experiment of this study was laid out in a factorial arrangement of $2 \times 2 \times 3$ in a completely randomized design (CRD) with three replications. These were three species of fish (tilapia, cat fish and common carp) with two types of preservatives (garlic, and ginger) and two methods of drying (oven drying and sun drying). The controls are fresh and dried fish fillets with no treatment.

Table 1 Experimental planning

\begin{tabular}{l|cccccc}
\hline \multirow{2}{*}{ Methods } & \multicolumn{6}{|c}{ Species } \\
\cline { 2 - 7 } & \multicolumn{2}{|c}{ Tilapia } & \multicolumn{2}{c}{ Cat fish } & \multicolumn{2}{c}{ Common carp } \\
\cline { 2 - 7 } & $\mathrm{Ga}$ & $\mathrm{Gi}$ & $\mathrm{Ga}$ & $\mathrm{Gi}$ & $\mathrm{Ga}$ & $\mathrm{Gi}$ \\
\hline $\mathrm{S}$ & $\mathrm{SGaT}$ & $\mathrm{SGiT}$ & $\mathrm{SGaC}$ & $\mathrm{SGiC}$ & $\mathrm{SGaCr}$ & $\mathrm{SGiCr}$ \\
$\mathrm{O}$ & OGaT & OGiT & OGaC & OGiC & OGaCr & OGiCr \\
Control & Fresh & Dried & fresh & Dried & fresh & Dried \\
\hline
\end{tabular}

Where: S (sun drying), O (oven drying), T (tilapia), C (cat fish), $\mathrm{Cr}$ (common carp), Ga (garlic), and Gi (ginger).

\section{Sample Preparation}

The sample preparation consists fish fillets preparation, ginger juice preparation, garlic juice preparation, pre-drying treatment of fish fillets, drying of fish fillets/samples and sun drying.

\section{Data Collection}

Data about moisture content, crude protein, crude fat, crude fiber, ash content, carbohydrate and gross energy collected from fresh and dried fillets of fish. Sensory evaluation of the dried fillets of fish conducted at the beginning on fresh, freshly dried and at one month interval for three months (AOAC, 1990).

\section{Experimental Design and Statistical Analysis}

Statistical analysis conducted on all data collected to test for significance difference among treatment means. Analysis of variance was performed by one-way ANOVA procedures with statistical software (version SAS 9.1) and means were evaluated at the $\mathrm{P}<0.05$ level of significance using fisher's LSD and Duncan's new multiple range test (AOAC, 2000).

\section{Result and Discussion}

The present study was conducted to evaluate the effect of sun drying, oven drying and two types of preservatives (garlic and ginger) on the nutritional value and sensory quality of tilapia (Oreochromis niloticus), cat fish (Pylodictis olivaris), and common carp (Cyprinus carpio) fish fillets.

\section{Fish}

The Effect of Drying on Proximate Composition of

The proximate composition of the fresh and dried fillets of the three species of fish namely cat fish, carp and tilapia are presented in Table 2. The moisture contents of the fresh cat fish, fresh carp and tilapia were 74.96, 75.81 and $74.74 \%$ respectively. These values were in agreement with those reported by Nurnadia et al (2011) which amount to $77.73 \%$ in Ostrea species.

The ash contents of the fresh and dried fillets of the three fish species are also shown in Table 2. The values are presented in dry basis and ranged from $6.34 \%$ of dried tilapia to $7.60 \%$ of dried carp. Significant $(\mathrm{P}<0.05)$ differences were noted among the values regardless of their moisture content. The ash contents recorded in fresh fillets namely cat fish, tilapia and carp 7.11, 6.50 and $7.59 \%$ respectively. No significant $(\mathrm{P}>0.05)$ variation was observed in ash content between the dry and wet fillets of carp.

Effect of Drying Methods on Proximate Composition
of Fish
The chemical composition of sun and oven dried fillets are presented in Table 3. The moisture contents of samples dried by the two methods were 8.77 and $7.17 \%$, respectively, with significant $(\mathrm{P}<0.05)$ difference between them.

The main effect of one of the factors, drying method, on the composition of the dried fillets in dry basis is shown in Table 3 . The ash content did not show significant $(\mathrm{P}<0.05)$ difference between samples dried in the sun and in the oven, with values of 7.08 and $7.10 \%$, respectively. Similar results of ash contents were reported by Oladipo and Bankole (2013) on dried tilapia, 7.28\%.

Effect of Preservatives on Proximate Composition of Dried Fillets.

Table 4 presents the effect of preservatives on composition of dried fillets. Samples treated with garlic and ginger had shown no difference in moisture content between them with values of 7.67 and $7.64 \%$ but are significantly lower than that of the control having $8.61 \%$ moisture.

The results in fat contents were laid within the values $6.55-11.02 \%$ reported by Oladipo and Bankole, (2013) in dried cat fish and tilapia species. The values of crude fat in this study were higher than those reported by Magawata and Shina (2013) with the fat content of $5.71 \%$. 
Table 2 Proximate composition of fresh and dried fillets of the three species

\begin{tabular}{l|cccccc}
\hline \multicolumn{1}{c|}{ Fish samples } & MC $(\%)$ & Ash $(\%$ db $)$ & Protein $(\% \mathrm{db})$ & Fat $(\% \mathrm{db})$ & Total carbs $(\% \mathrm{db})$ & Gross energy $(\mathrm{kcal} / 100 \mathrm{~g})$ \\
\hline Fresh cat fish & $74.96 \pm 0.00^{\mathrm{b}}$ & $7.11 \pm 0.04^{\mathrm{c}}$ & $75.90 \pm 0.01^{\mathrm{b}}$ & $8.52 \pm 0.13^{\mathrm{b}}$ & $8.47 \pm 0.13^{\mathrm{bc}}$ & $414.15 \pm 0.69^{\mathrm{ba}}$ \\
Fresh carp & $75.81 \pm 0.39^{\mathrm{a}}$ & $7.59 \pm 0.06^{\mathrm{a}}$ & $74.20 \pm 0.20^{\mathrm{c}}$ & $9.09 \pm 0.14^{\mathrm{a}}$ & $9.12 \pm 0.17^{\mathrm{ba}}$ & $415.10 \pm 0.67^{\mathrm{a}}$ \\
Fresh tilapia & $74.74 \pm 0.11^{\mathrm{b}}$ & $6.50 \pm 0.04^{\mathrm{d}}$ & $76.67 \pm 0.33^{\mathrm{a}}$ & $8.06 \pm 0.16^{\mathrm{c}}$ & $8.77 \pm 0.50^{\mathrm{bac}}$ & $414.28 \pm 0.63^{\mathrm{ba}}$ \\
Dried cat fish & $7.91 \pm 0.12^{\mathrm{c}}$ & $7.34 \pm 0.05^{\mathrm{b}}$ & $75.84 \pm 0.02^{\mathrm{b}}$ & $8.53 \pm 0.01^{\mathrm{b}}$ & $8.29 \pm 0.03^{\mathrm{c}}$ & $413.28 \pm 0.23^{\mathrm{b}}$ \\
Dried carp & $7.76 \pm 0.02^{\mathrm{c}}$ & $7.60 \pm 0.02^{\mathrm{a}}$ & $74.08 \pm 0.02^{\mathrm{c}}$ & $9.13 \pm 0.00^{\mathrm{a}}$ & $9.19 \pm 0.01^{\mathrm{a}}$ & $415.26 \pm 0.07^{\mathrm{a}}$ \\
Dried tilapia & $8.25 \pm 0.09^{\mathrm{c}}$ & $6.34 \pm 0.01^{\mathrm{e}}$ & $76.74 \pm 0.02^{\mathrm{a}}$ & $8.11 \pm 0.01^{\mathrm{c}}$ & $8.81 \pm 0.01^{\mathrm{bac}}$ & $415.22 \pm 0.03^{\mathrm{a}}$ \\
CV & 0.74 & 0.97 & 0.36 & 2.09 & 4.40 & 0.20 \\
LSD & 0.55 & 0.12 & 0.48 & 0.32 & 0.69 & 1.47 \\
\hline
\end{tabular}

Where, $\mathrm{LSD}=$ list significant difference, $\mathrm{CV}=$ coefficient of variation; $\mathrm{MC}=$ moisture content and the values are mean $\pm \mathrm{SE}$ in that the mean values followed by the same letter in a column are not significantly different at $5 \%$ level of significance. Gross energy is computed per $100 \mathrm{~g}$ dry sample.

Table 3 Proximate composition of dried fillets of the two method of drying

\begin{tabular}{l|cccccc}
\hline \multicolumn{1}{c|}{ MD } & MC $(\%)$ & Ash $(\%, \mathrm{db})$ & Protein $(\% \mathrm{db})$ & Fat $(\%, \mathrm{db})$ & Total carbs $(\%$ db) & Gross energy $(\mathrm{kcal} / 100 \mathrm{~g})$ \\
\hline Oven drying & $7.17 \pm 0.05^{\mathrm{b}}$ & $7.10 \pm 0.12^{\mathrm{a}}$ & $75.78 \pm 0.19^{\mathrm{a}}$ & $7.75 \pm 0.14^{\mathrm{b}}$ & $9.37 \pm 0.17^{\mathrm{a}}$ & $410.35 \pm 0.73^{\mathrm{b}}$ \\
Sun drying & $8.77 \pm 0.22^{\mathrm{a}}$ & $7.08 \pm 0.20^{\mathrm{a}}$ & $75.35 \pm 0.33^{\mathrm{a}}$ & $9.44 \pm 0.12^{\mathrm{a}}$ & $8.13 \pm 0.17^{\mathrm{b}}$ & $418.92 \pm 0.64^{\mathrm{a}}$ \\
CV & 10.24 & 11.92 & 1.83 & 7.94 & 10.20 & 0.86 \\
LSD & 0.45 & 0.46 & 0.76 & 0.37 & 0.49 & 1.95 \\
\hline
\end{tabular}

Where, $\mathrm{MD}=$ method of drying, $\mathrm{LSD}=$ list significant difference, $\mathrm{CV}=$ coefficient of variation; $\mathrm{MC}=$ moisture content and the values are mean $\pm \mathrm{SE}$ in that the mean values followed by the same letter in a column are not significantly different at $5 \%$ level of significance.

Table 4 Proximate composition of the dried fillets subjected to preservatives

\begin{tabular}{l|cccccc}
\hline \multicolumn{1}{c|}{ TR } & MC $(\%)$ & Ash $(\% \mathrm{db})$ & Protein $(\% \mathrm{db})$ & Fat $(\% \mathrm{db})$ & Total carbs $(\% \mathrm{db})$ & Gross energy $(\mathrm{kcal} / 100 \mathrm{~g})$ \\
\hline Control & $8.61 \pm 0.40^{\mathrm{a}}$ & $7.24 \pm 0.17^{\mathrm{a}}$ & $75.37 \pm 0.22^{\mathrm{a}}$ & $8.50 \pm 0.33^{\mathrm{ba}}$ & $8.89 \pm 0.34^{\mathrm{a}}$ & $413.54 \pm 1.64^{\mathrm{a}}$ \\
Garlic & $7.67 \pm 0.09^{\mathrm{b}}$ & $7.15 \pm 0.19^{\mathrm{a}}$ & $75.41 \pm 0.30^{\mathrm{a}}$ & $9.07 \pm 0.18^{\mathrm{a}}$ & $8.37 \pm 0.17^{\mathrm{a}}$ & $416.70 \pm 0.94^{\mathrm{a}}$ \\
Ginger & $7.64 \pm 0.15^{\mathrm{b}}$ & $6.88 \pm 0.23^{\mathrm{a}}$ & $75.91 \pm 0.43^{\mathrm{a}}$ & $8.23 \pm 0.21^{\mathrm{b}}$ & $8.98 \pm 0.22^{\mathrm{a}}$ & $413.68 \pm 1.21^{\mathrm{a}}$ \\
CV & 13.40 & 11.82 & 1.85 & 12.25 & 12.24 & 1.32 \\
LSD & 0.71 & 0.56 & 0.93 & 0.71 & 0.72 & 3.67 \\
\hline
\end{tabular}

Where, $\mathrm{TR}=$ Treatment, $\mathrm{LSD}=$ list significant difference, $\mathrm{CV}=$ coefficient of variation; $\mathrm{MC}=$ moisture content and the values are mean $\pm \mathrm{SE}$ in that the mean values followed by the same letter in a column are not significantly different at $5 \%$ level of significance.

Table 5 Composition of preservatives interaction with methods of drying on dried fillets

\begin{tabular}{c|lcccccc}
\hline MD & TR & MC $(\%)$ & Ash $(\% \mathrm{db})$ & Protein $(\% \mathrm{db})$ & Fat $(\% \mathrm{db})$ & Total carbs $(\% \mathrm{db})$ & Gross energy $(\mathrm{kcal} / 100 \mathrm{~g})$ \\
\hline \multirow{3}{*}{$\mathrm{SD}$} & $\mathrm{S}$ & $10.18 \pm 0.28^{\mathrm{a}}$ & $7.31 \pm 0.29^{\mathrm{ba}}$ & $75.10 \pm 0.33^{\mathrm{a}}$ & $9.71 \pm 0.13^{\mathrm{a}}$ & $7.88 \pm 0.30^{\mathrm{c}}$ & $419.33 \pm 1.46^{\mathrm{a}}$ \\
& $\mathrm{Ga}$ & $7.91 \pm 0.07^{\mathrm{b}}$ & $7.36 \pm 0.35^{\mathrm{a}}$ & $74.84 \pm 0.49^{\mathrm{a}}$ & $9.75 \pm 0.06^{\mathrm{a}}$ & $8.05 \pm 0.27^{\mathrm{cb}}$ & $419.30 \pm 1.13^{\mathrm{a}}$ \\
& $\mathrm{Gi}$ & $8.23 \pm 0.05^{\mathrm{b}}$ & $6.56 \pm 0.36^{\mathrm{b}}$ & $76.12 \pm 0.76^{\mathrm{a}}$ & $8.88 \pm 0.04^{\mathrm{b}}$ & $8.44 \pm 0.33^{\mathrm{cb}}$ & $418.15 \pm 0.75^{\mathrm{a}}$ \\
\hline & $\mathrm{O}$ & $7.05 \pm 0.01^{\mathrm{d}}$ & $7.16 \pm 0.19^{\mathrm{ba}}$ & $75.65 \pm 0.27^{\mathrm{a}}$ & $7.28 \pm 0.26^{\mathrm{c}}$ & $9.91 \pm 0.37^{\mathrm{a}}$ & $407.76 \pm 0.94^{\mathrm{c}}$ \\
OD & $\mathrm{Ga}$ & $7.43 \pm 0.11^{\mathrm{c}}$ & $6.95 \pm 0.13^{\mathrm{ba}}$ & $75.98 \pm 0.25^{\mathrm{a}}$ & $8.38 \pm 0.15^{\mathrm{b}}$ & $8.69 \pm 0.16^{\mathrm{b}}$ & $414.10 \pm 0.91^{\mathrm{b}}$ \\
& $\mathrm{Gi}$ & $7.05 \pm 0.01^{\mathrm{d}}$ & $7.19 \pm 0.28^{\mathrm{ba}}$ & $75.70 \pm 0.46^{\mathrm{a}}$ & $7.60 \pm 0.17^{\mathrm{c}}$ & $9.51 \pm 0.16^{\mathrm{a}}$ & $409.20 \pm 0.81^{\mathrm{c}}$ \\
\hline CV & & 4.73 & 11.72 & 1.82 & 6.31 & 9.48 & 0.74 \\
LSD & & 0.36 & 0.79 & 1.31 & 0.51 & 0.79 & 2.91 \\
\hline
\end{tabular}

Where, $\mathrm{SO}=$ sun drying, $\mathrm{OD}=$ oven drying, $\mathrm{TR}=$ treatment, $\mathrm{S}=$ sundrying, $\mathrm{Ga}=$ garlic,Gi= ginger, $\mathrm{O}=$ oven drying, $\mathrm{LSD}=$ list significant difference, $\mathrm{CV}=$ coefficient of variation; $\mathrm{MC}=$ moisture content and $\mathrm{MD}=$ method of drying.

Table 6 Composition of fish species interaction with method of drying on dried fish fillets

\begin{tabular}{l|lcccccc}
\hline MD & Spp. & MC $(\%)$ & Ash $(\% \mathrm{db})$ & Protein $(\% \mathrm{db})$ & Fat $(\% \mathrm{db})$ & Total carbs $(\% \mathrm{db})$ & Gross energy (kcal/100g) \\
\hline \multirow{3}{*}{ SD } & $\mathrm{T}$ & $9.20 \pm 0.49^{\mathrm{a}}$ & $6.13 \pm 0.13^{\mathrm{b}}$ & $77.03 \pm 0.40^{\mathrm{a}}$ & $9.12 \pm 0.23^{\mathrm{b}}$ & $7.72 \pm 0.19^{\mathrm{d}}$ & $421.08 \pm 1.10^{\mathrm{a}}$ \\
& $\mathrm{C}$ & $8.78 \pm 0.35^{\mathrm{ba}}$ & $7.33 \pm 0.39^{\mathrm{a}}$ & $75.58 \pm 0.20^{\mathrm{c}}$ & $9.31 \pm 0.20^{\mathrm{b}}$ & $7.78 \pm 0.38^{\mathrm{d}}$ & $417.23 \pm 1.04^{\mathrm{b}}$ \\
& $\mathrm{Cr}$ & $8.34 \pm 0.23^{\mathrm{b}}$ & $7.77 \pm 0.17^{\mathrm{a}}$ & $73.45 \pm 0.15^{\mathrm{e}}$ & $9.91 \pm 0.06^{\mathrm{a}}$ & $8.87 \pm 0.05^{\mathrm{bc}}$ & $418.47 \pm 0.88^{\mathrm{ba}}$ \\
\hline & $\mathrm{T}$ & $7.30 \pm 0.13^{\mathrm{c}}$ & $6.52 \pm 0.11^{\mathrm{b}}$ & $76.48 \pm 0.22^{\mathrm{ba}}$ & $7.09 \pm 0.23^{\mathrm{e}}$ & $9.91 \pm 0.36^{\mathrm{a}}$ & $409.33 \pm 1.24^{\mathrm{c}}$ \\
OD & $\mathrm{C}$ & $7.04 \pm 0.01^{\mathrm{c}}$ & $7.34 \pm 0.18^{\mathrm{a}}$ & $76.13 \pm 0.20^{\mathrm{bc}}$ & $7.79 \pm 0.17^{\mathrm{d}}$ & $8.74 \pm 0.16^{\mathrm{c}}$ & $409.63 \pm 1.27^{\mathrm{c}}$ \\
& $\mathrm{Cr}$ & $7.18 \pm 0.06^{\mathrm{c}}$ & $7.44 \pm 0.17^{\mathrm{a}}$ & $74.71 \pm 0.21^{\mathrm{d}}$ & $8.37 \pm 0.12^{\mathrm{c}}$ & $9.48 \pm 0.21^{\text {ba }}$ & $412.10 \pm 1.49^{\mathrm{c}}$ \\
\hline CV & & 10.08 & 9.02 & 0.97 & 6.22 & 8.66 & 0.82 \\
LSD & & 0.76 & 0.61 & 0.69 & 0.51 & 0.72 & 3.21 \\
\hline
\end{tabular}

Where, $\mathrm{SD}=$ sundrying, $\mathrm{OD}=$ ovendrying, $\mathrm{LSD}=$ list significant difference, $\mathrm{CV}=$ coefficient of variation, $\mathrm{MC}=$ moisture content, $\mathrm{MD}=$ method of drying, Spp.=species, $\mathrm{T}=$ tilapia, $\mathrm{C}=$ cat fish, $\mathrm{Cr}=$ common carp and the values are mean $\pm \mathrm{SE}$ in that the mean values followed by the same letter in a column are not significantly different at $5 \%$ level of significance. 
Table 7 Data showing the interaction effect of preservatives and fish spp. on composition

\begin{tabular}{l|lcccccc}
\hline Treatment & Spp. & MC $(\%)$ & Ash $(\% \mathrm{db})$ & Protein $(\% \mathrm{db})$ & Fat $(\% \mathrm{db})$ & Total carbs $(\% \mathrm{db})$ & $\mathrm{Gross} e n e r g y(k c a l / 100 \mathrm{~g})$ \\
\hline \multirow{3}{*}{ Control } & $\mathrm{T}$ & $9.08 \pm 0.92^{\mathrm{a}}$ & $6.55 \pm 0.14^{\mathrm{dc}}$ & $75.89 \pm 0.10^{\mathrm{b}}$ & $7.98 \pm 0.72^{\mathrm{bc}}$ & $9.58 \pm 0.77^{\mathrm{a}}$ & $413.70 \pm 3.85^{\mathrm{ba}}$ \\
& $\mathrm{C}$ & $8.61 \pm 0.70^{\mathrm{ba}}$ & $7.73 \pm 0.30^{\mathrm{a}}$ & $75.87 \pm 0.29^{\mathrm{b}}$ & $8.41 \pm 0.49^{\mathrm{bac}}$ & $7.99 \pm 0.49^{\mathrm{dc}}$ & $411.15 \pm 1.74^{\mathrm{b}}$ \\
& $\mathrm{Cr}$ & $8.14 \pm 0.48^{\mathrm{ba}}$ & $7.43 \pm 0.15^{\mathrm{ba}}$ & $74.36 \pm 0.27^{\mathrm{c}}$ & $9.10 \pm 0.44^{\mathrm{ba}}$ & $9.11 \pm 0.24^{\mathrm{ba}}$ & $415.79 \pm 2.71^{\mathrm{ba}}$ \\
\hline \multirow{3}{*}{ Garlic } & $\mathrm{T}$ & $7.93 \pm 0.06^{\mathrm{ba}}$ & $6.37 \pm 0.20^{\mathrm{dc}}$ & $76.45 \pm 0.08^{\mathrm{b}}$ & $8.70 \pm 0.38^{\mathrm{bac}}$ & $8.48 \pm 0.20^{\mathrm{bdac}}$ & $418.03 \pm 2.64^{\mathrm{a}}$ \\
& $\mathrm{C}$ & $7.54 \pm 0.23^{\mathrm{b}}$ & $7.49 \pm 0.20^{\mathrm{a}}$ & $75.69 \pm 0.32^{\mathrm{b}}$ & $9.14 \pm 0.31^{\mathrm{ba}}$ & $7.68 \pm 0.27^{\mathrm{d}}$ & $415.77 \pm 0.98^{\mathrm{ba}}$ \\
& $\mathrm{Cr}$ & $7.54 \pm 0.05^{\mathrm{b}}$ & $7.60 \pm 0.29^{\mathrm{a}}$ & $74.09 \pm 0.49^{\mathrm{c}}$ & $9.34 \pm 0.25^{\mathrm{a}}$ & $8.97 \pm 0.13^{\mathrm{bac}}$ & $416.29 \pm 0.78^{\mathrm{ba}}$ \\
\hline \multirow{3}{*}{ Ginger } & $\mathrm{T}$ & $7.74 \pm 0.30^{\mathrm{b}}$ & $6.07 \pm 0.09^{\mathrm{d}}$ & $77.92 \pm 0.32^{\mathrm{a}}$ & $7.63 \pm 0.29^{\mathrm{c}}$ & $8.38 \pm 0.55^{\mathrm{bcc}}$ & $413.89 \pm 1.75^{\mathrm{ba}}$ \\
& $\mathrm{C}$ & $7.58 \pm 0.24^{\mathrm{b}}$ & $6.78 \pm 0.47^{\mathrm{bc}}$ & $76.02 \pm 0.18^{\mathrm{b}}$ & $8.10 \pm 0.24^{\mathrm{bc}}$ & $9.10 \pm 0.10^{\mathrm{bac}}$ & $413.37 \pm 3.00^{\mathrm{ba}}$ \\
& $\mathrm{Cr}$ & $7.60 \pm 0.25^{\mathrm{b}}$ & $7.78 \pm 0.16^{\mathrm{a}}$ & $73.79 \pm 0.23^{\mathrm{c}}$ & $8.98 \pm 0.35^{\mathrm{ba}}$ & $9.45 \pm 0.25^{\mathrm{ba}}$ & $413.77 \pm 1.66^{\mathrm{ba}}$ \\
\hline CV & & 13.85 & 8.61 & 0.91 & 11.74 & 11.03 & 1.37 \\
LSD & & 1.28 & 0.71 & 0.80 & 1.17 & 1.12 & 6.61 \\
\hline
\end{tabular}

Where, LSD=list significant difference, $\mathrm{CV}=$ coefficient of variation, $\mathrm{MC}=$ moisture content, $\mathrm{MD}=$ method of drying, Spp.=species, $\mathrm{T}=$ tilapia, $\mathrm{C}=\mathrm{cat}$ fish, $\mathrm{Cr}=$ common carp and the values are mean $\pm \mathrm{SE}$ in that the mean values followed by the same letter in a column are not significantly different at $5 \%$ level of significance.

Table 8 Proximate composition of differently treated dried fillets before storage

\begin{tabular}{|c|c|c|c|c|c|c|c|c|}
\hline MD & TR & Spp. & $\mathrm{MC}(\%)$ & $\operatorname{Ash}(\% \mathrm{db})$ & Protein $(\% \mathrm{db})$ & Fat $(\% \mathrm{db})$ & Total carbs $(\% \mathrm{db})$ & Gross energy $(\mathrm{kcal} / 100 \mathrm{~g})$ \\
\hline \multirow{9}{*}{ Sun drying } & \multirow{3}{*}{ Control } & $\mathrm{T}$ & $11.13 \pm 0.02^{\mathrm{a}}$ & $6.43 \pm 0.28^{\text {hfg }}$ & $76.09 \pm 0.11^{\mathrm{dce}}$ & $9.59 \pm 0.03^{\mathrm{bdc}}$ & $7.89 \pm 0.33^{\mathrm{e}}$ & $422.21 \pm 1.10^{\mathrm{ba}}$ \\
\hline & & $\mathrm{C}$ & $10.17 \pm 0.01^{\mathrm{b}}$ & $8.31 \pm 0.12^{\mathrm{a}}$ & $75.32 \pm 0.07^{\mathrm{gfh}}$ & $9.47 \pm 0.29^{\mathrm{d}}$ & $6.90 \pm 0.11^{\mathrm{f}}$ & $414.13 \pm 1.95^{\text {fge }}$ \\
\hline & & $\mathrm{Cr}$ & $9.23 \pm 0.02^{\mathrm{c}}$ & $7.19 \pm 0.06^{\mathrm{edc}}$ & $73.88 \pm 0.08^{\mathrm{ji}}$ & $10.08 \pm 0.08^{\mathrm{a}}$ & $8.85 \pm 0.00^{\mathrm{d}}$ & $421.65 \pm 0.57^{\text {ba }}$ \\
\hline & \multirow{3}{*}{ Garlic } & $\mathrm{T}$ & $8.05 \pm 0.00^{f}$ & $6.01 \pm 0.19^{\mathrm{hi}}$ & $76.37 \pm 0.10^{\mathrm{c}}$ & $9.52 \pm 0.01^{\mathrm{dc}}$ & $8.10 \pm 0.24^{\mathrm{e}}$ & $423.56 \pm 0.80^{\mathrm{a}}$ \\
\hline & & $\mathrm{C}$ & $8.05 \pm 0.03^{\mathrm{f}}$ & $7.89 \pm 0.07^{\text {ba }}$ & $75.12 \pm 0.10^{\mathrm{gh}}$ & $9.84 \pm 0.06^{\mathrm{bac}}$ & $7.15 \pm 0.12^{\mathrm{f}}$ & $417.63 \pm 0.58^{\mathrm{dc}}$ \\
\hline & & $\mathrm{Cr}$ & $7.64 \pm 0.01^{\mathrm{h}}$ & $8.18 \pm 0.18^{\text {ba }}$ & $73.03 \pm 0.25^{\mathrm{k}}$ & $9.89 \pm 0.06^{\mathrm{ba}}$ & $8.90 \pm 0.17^{\mathrm{d}}$ & $416.70 \pm 0.63^{\mathrm{dfce}}$ \\
\hline & \multirow{3}{*}{ Ginger } & $\mathrm{T}$ & $8.42 \pm 0.01^{\mathrm{d}}$ & $5.95 \pm 0.16^{\mathrm{hi}}$ & $78.62 \pm 0.10^{\mathrm{a}}$ & $8.25 \pm 0.21^{\mathrm{g}}$ & $7.18 \pm 0.24^{f}$ & $417.46 \pm 1.54^{\mathrm{dce}}$ \\
\hline & & $\mathrm{C}$ & $8.13 \pm 0.01^{\mathrm{e}}$ & $5.79 \pm 0.18^{\mathrm{i}}$ & $76.31 \pm 0.2^{\mathrm{dc}}$ & $8.62 \pm 0.08^{\mathrm{fe}}$ & $9.28 \pm 0.03^{\mathrm{cd}}$ & $419.93 \pm 0.66^{\mathrm{bc}}$ \\
\hline & & $\mathrm{Cr}$ & $8.16 \pm 0.01^{\mathrm{e}}$ & $7.94 \pm 0.17^{\text {ba }}$ & $73.42 \pm 0.11^{\mathrm{jk}}$ & $9.76 \pm 0.09^{\mathrm{bdac}}$ & $8.88 \pm 0.04^{\mathrm{d}}$ & $417.06 \pm 1.01^{\mathrm{dfce}}$ \\
\hline \multirow{9}{*}{ Oven drying } & \multirow{3}{*}{ Control } & $\mathrm{T}$ & $7.03 \pm 0.01^{j}$ & $6.67 \pm 0.09^{\mathrm{efg}}$ & $75.70 \pm 0.04^{\mathrm{gfe}}$ & $6.37 \pm 0.07^{1}$ & $11.26 \pm 0.12^{\mathrm{a}}$ & $405.19 \pm 0.66^{1}$ \\
\hline & & $\mathrm{C}$ & $7.06 \pm 0.01^{\mathrm{j}}$ & $7.16 \pm 0.33^{\text {ed }}$ & $76.41 \pm 0.35^{\mathrm{c}}$ & $7.36 \pm 0.07^{\mathrm{j}}$ & $9.07 \pm 0.08^{\mathrm{cd}}$ & $408.16 \pm 1.56^{\mathrm{kjl}}$ \\
\hline & & $\mathrm{Cr}$ & $7.06 \pm 0.02^{j}$ & $7.66 \pm 0.25^{\mathrm{bdc}}$ & $74.84 \pm 0.37^{\mathrm{h}}$ & $8.12 \pm 0.10^{\text {hg }}$ & $9.38 \pm 0.48^{\mathrm{cd}}$ & $409.94 \pm 1.41^{\mathrm{kji}}$ \\
\hline & \multirow{3}{*}{ Garlic } & $\mathrm{T}$ & $7.80 \pm 0.00^{\mathrm{g}}$ & $6.72 \pm 0.21^{\mathrm{efg}}$ & $76.53 \pm 0.12^{\mathrm{c}}$ & $7.88 \pm 0.21^{\mathrm{hi}}$ & $8.87 \pm 0.04^{\mathrm{d}}$ & $412.50 \pm 1.88^{\mathrm{hgi}}$ \\
\hline & & $\mathrm{C}$ & $7.04 \pm 0.02^{\mathrm{j}}$ & $7.09 \pm 0.20^{\text {ed }}$ & $76.26 \pm 0.42^{\text {dce }}$ & $8.45 \pm 0.05^{\mathrm{fg}}$ & $8.20 \pm 0.26^{\mathrm{e}}$ & $413.92 \pm 1.02^{\mathrm{hfg}}$ \\
\hline & & $\mathrm{Cr}$ & $7.44 \pm 0.00^{\mathrm{i}}$ & $7.03 \pm 0.26^{\mathrm{ef}}$ & $75.15 \pm 0.19^{\mathrm{gfh}}$ & $8.80 \pm 0.11^{\mathrm{e}}$ & $9.02 \pm 0.22^{\mathrm{d}}$ & $415.88 \pm 1.56^{\mathrm{dfge}}$ \\
\hline & \multirow{3}{*}{ Ginger } & $\mathrm{T}$ & $7.06 \pm 0.01^{j}$ & $6.19 \pm 0.08^{\text {hig }}$ & $77.22 \pm 0.02^{b}$ & $7.01 \pm 0.04^{\mathrm{k}}$ & $9.58 \pm 0.02^{\mathrm{cb}}$ & $410.31 \pm 0.49^{\mathrm{ji}}$ \\
\hline & & $\mathrm{C}$ & $7.04 \pm 0.01^{\mathrm{j}}$ & $7.76 \pm 0.30^{\text {bac }}$ & $75.73 \pm 0.17^{\mathrm{dfe}}$ & $7.58 \pm 0.01^{\mathrm{ji}}$ & $8.93 \pm 0.12^{\mathrm{d}}$ & $406.82 \pm 1.23^{\mathrm{kl}}$ \\
\hline & & $\mathrm{Cr}$ & $7.05 \pm 0.01^{\mathrm{j}}$ & $7.63 \pm 0.28^{\mathrm{bdc}}$ & $74.15 \pm 0.33^{\mathrm{i}}$ & $8.20 \pm 0.10^{\text {hg }}$ & $10.02 \pm 0.07^{\mathrm{b}}$ & $410.47 \pm 1.38^{\mathrm{hji}}$ \\
\hline $\mathrm{CV}$ & & & 0.27 & 5.03 & 0.48 & 2.38 & 3.79 & 0.50 \\
\hline LSD & & & 0.04 & 0.59 & 0.61 & 0.34 & 0.55 & 3.45 \\
\hline
\end{tabular}

Where, TR=Treatment, $\mathrm{LSD}=$ list significant difference, $\mathrm{CV}=$ coefficient of variation; $\mathrm{MC}=$ moisture content, $\mathrm{MD}=$ method of drying, Spp.=species, $\mathrm{T}=$ tilapia, $\mathrm{C}=$ cat fish, $\mathrm{Cr}=$ common carp and the values are mean $\pm \mathrm{SE}$ in that the mean values followed by the same letter in a column are not significantly different at $5 \%$ level of significance.

Effect of Interaction between Drying Methods and Preservatives on Composition

Table 5 presents data showing the interaction effect of drying method and preservative treatments on the proximate composition. The drying methods interacted with preservatives significantly $(\mathrm{P}<0.05)$ affecting the moisture content of dried fillets. The highest $(10.18 \%)$ moisture content was recorded for untreated sun dried fillets and the next two higher values belongs to ginger treated and garlic treated sundried samples with 8.23 and $7.91 \%$ showing no statistical difference between them.

It can easily be seen that the majority of the data do not have statistical differences staying between $7.36 \%$ of the garlic treated sundried sample and $6.56 \%$ of the ginger treated sample dried in the sun. Only these two values exhibit significant difference in ash at $\mathrm{P}<0.05$.

Effect of Interaction between Drying Methods and Fish Species on Composition

Data of the interactions between the drying methods of fillets with fish species is presented in Table 6. The interaction significantly $(\mathrm{P}<0.05)$ affected the moisture content of dried fillets. Differences in moisture content of the fillets were observed due to the different treatment combinations. The highest three moisture contents were recorded for sun dried fillet of the three species with values of $9.20,8.78$ and $8.34 \%$ for tilapia, cat fish and carp respectively.

The interaction of the two factors also affected ash contents with significant $(\mathrm{P}<0.05)$ difference between tilapia and the two other species. Both fillets of the former dried in the sun and oven had lower values, 6.13 and $6.52 \%$ respectively than fillets of the latter dried in both sun and oven.

\section{Effect of Interaction between Preservatives and} Species of Fish on Composition.

Table 7 presents the proximate composition data of the dried fillets as affected by the interaction of preservatives and fish species. Not much significant $(\mathrm{P}>0.05)$ differences have been noted among the values as most values were followed by same letter as superscript. The control samples of all three fish species exhibited relatively higher moisture content showing the preservatives did not contribute to lower moisture contents in all the three species. 
The contents of carbohydrate varied from 7.68 to of garlic treated cat fish to $9.58 \%$ of tilapia used as control. The interaction of preservatives treatments with species resulted in significant $(\mathrm{P}<0.05)$ differences among the samples, in respect of their carbohydrate content, including the highest and lowest values indicated above.

Proximate Composition of Differently Treated Dried Fillets before Storage

The data about proximate composition of dried fillets before storage was presented in Table 8 . The moisture contents of the sun dried samples which were not treated with ginger or garlic were between 9.23 and 11.13 percent. These values are significantly $(\mathrm{P}<0.05)$ higher than those of the other samples treated with ginger or garlic including all oven dried samples. Further almost all the sun dried garlic and ginger treated samples exhibited higher moisture contents (7.64 up to $8.16 \%$ ) than those of the oven dried samples which remained less than $7.10 \%$ except that of the garlic treated tilapia with 7.80 percent.

\section{Composition of Differently Treated Dried Fillets after} One Month Storage

Proximate composition of one month stored products of differently treated dried fillets of the three fish species namely tilapia, cat fish and carp presented in Table 9. The results obtained from the present study revealed that significant $(\mathrm{P}<0.05)$ differences in moisture contents observed among the untreated sundried and treated (7.33$9.09 \%$ ) fillets of the dried fish. In this experiment the percentages of moisture contents of the dried fillets were increased after the storage period of one month. This may due to the re-absorption of the residual moisture by the dried fillets while in storage (Ames et al., 1991).

Table 9 Proximate composition of differently treated dried fillets after the storage of one month

\begin{tabular}{|c|c|c|c|c|c|c|c|c|}
\hline MD & TR & Spp. & $\mathrm{MC}(\%)$ & $\mathrm{Ash}(\% \mathrm{db})$ & Protein $(\% \mathrm{db})$ & Fat $(\% \mathrm{db})$ & Total carbs $(\% \mathrm{db})$ & Gross energy $(\mathrm{kcal} / 100 \mathrm{~g})$ \\
\hline \multirow{9}{*}{ Sun drying } & \multirow{3}{*}{ Control } & $\mathrm{T}$ & $11.80 \pm 0.02^{\mathrm{a}}$ & $6.35 \pm 0.28^{\text {ihg }}$ & $76.26 \pm 0.11^{\mathrm{dce}}$ & $9.59 \pm 0.03^{\mathrm{bc}}$ & $7.80 \pm 0.33^{f}$ & $422.58 \pm 1.11^{\mathrm{a}}$ \\
\hline & & $\mathrm{C}$ & $11.06 \pm 0.01^{\mathrm{b}}$ & $8.07 \pm 0.12^{\text {ba }}$ & $75.59 \pm 0.07^{\mathrm{gfh}}$ & $9.45 \pm 0.30^{c}$ & $6.89 \pm 0.11^{\mathrm{g}}$ & $414.97 \pm 1.96^{\mathrm{ef}}$ \\
\hline & & $\mathrm{Cr}$ & $9.51 \pm 0.02^{\mathrm{c}}$ & $7.19 \pm 0.06^{\text {edc }}$ & $73.95 \pm 0.08^{\mathrm{kj}}$ & $10.04 \pm 0.08^{\mathrm{a}}$ & $8.82 \pm 0.00^{\mathrm{de}}$ & $421.42 \pm 0.57^{\text {ba }}$ \\
\hline & \multirow{3}{*}{ Garlic } & $\mathrm{T}$ & $8.72 \pm 0.00^{\mathrm{g}}$ & $5.92 \pm 0.19^{\mathrm{ij}}$ & $76.54 \pm 0.10^{\mathrm{dc}}$ & $9.52 \pm 0.01^{\mathrm{bc}}$ & $8.02 \pm 0.24^{f}$ & $423.93 \pm 0.81^{\mathrm{a}}$ \\
\hline & & $\mathrm{C}$ & $8.94 \pm 0.03^{\mathrm{f}}$ & $7.66 \pm 0.07^{\mathrm{bac}}$ & $75.38 \pm 0.10^{\text {gih }}$ & $9.82 \pm 0.06^{\text {ba }}$ & $7.14 \pm 0.12^{\mathrm{g}}$ & $418.48 \pm 0.59^{\mathrm{bdc}}$ \\
\hline & & $\mathrm{Cr}$ & $7.92 \pm 0.01^{\mathrm{i}}$ & $8.18 \pm 0.18^{\mathrm{a}}$ & $73.09 \pm 0.25^{1}$ & $9.84 \pm 0.06^{\text {ba }}$ & $8.89 \pm 0.17^{\mathrm{de}}$ & $416.46 \pm 0.64^{\mathrm{ed}}$ \\
\hline & \multirow{3}{*}{ Ginger } & $\mathrm{T}$ & $9.09 \pm 0.01^{\mathrm{d}}$ & $5.86 \pm 0.16^{\mathrm{ij}}$ & $78.80 \pm 0.10^{\mathrm{a}}$ & $8.24 \pm 0.21^{\mathrm{ef}}$ & $7.10 \pm 0.24^{\mathrm{g}}$ & $417.79 \pm 1.55^{\text {edc }}$ \\
\hline & & $\mathrm{C}$ & $9.02 \pm 0.01^{\mathrm{e}}$ & $5.54 \pm 0.18^{\mathrm{j}}$ & $76.58 \pm 0.21^{\mathrm{dc}}$ & $8.59 \pm 0.08^{\mathrm{d}}$ & $9.29 \pm 0.03^{\text {cde }}$ & $420.80 \pm 0.67^{\text {bac }}$ \\
\hline & & $\mathrm{Cr}$ & $8.44 \pm 0.01^{\mathrm{h}}$ & $7.94 \pm 0.17^{\text {ba }}$ & $73.48 \pm 0.11^{\mathrm{kl}}$ & $9.71 \pm 0.09^{\mathrm{bac}}$ & $8.87 \pm 0.04^{\mathrm{de}}$ & $416.82 \pm 1.02^{\text {ed }}$ \\
\hline \multirow{9}{*}{ Oven drying } & \multirow{3}{*}{ Control } & $\mathrm{T}$ & $7.70 \pm 0.01^{j}$ & $6.58 \pm 0.09^{\mathrm{hgf}}$ & $75.86 \pm 0.04^{\mathrm{gfe}}$ & $6.35 \pm 0.07^{\mathrm{k}}$ & $11.21 \pm 0.12^{\mathrm{a}}$ & $405.42 \pm 0.66^{i}$ \\
\hline & & $\mathrm{C}$ & $7.95 \pm 0.01^{\mathrm{i}}$ & $6.92 \pm 0.34^{\mathrm{egf}}$ & $76.68 \pm 0.36^{\mathrm{c}}$ & $7.32 \pm 0.07^{\mathrm{ij}}$ & $9.08 \pm 0.08^{\text {cde }}$ & $408.91 \pm 1.58^{\mathrm{h}}$ \\
\hline & & $\mathrm{Cr}$ & $7.34 \pm 0.02^{\mathrm{k}}$ & $7.66 \pm 0.25^{\mathrm{bac}}$ & $74.91 \pm 0.37^{\mathrm{i}}$ & $8.06 \pm 0.10^{\mathrm{fg}}$ & $9.37 \pm 0.48^{\mathrm{cd}}$ & $409.68 \pm 1.41^{\mathrm{hg}}$ \\
\hline & \multirow{3}{*}{ Garlic } & $\mathrm{T}$ & $8.47 \pm 0.00^{h}$ & $6.64 \pm 0.21^{\text {ehgf }}$ & $76.70 \pm 0.12^{\mathrm{c}}$ & $7.87 \pm 0.21^{\text {hg }}$ & $8.79 \pm 0.04^{\mathrm{e}}$ & $412.79 \pm 1.89^{\mathrm{gf}}$ \\
\hline & & $\mathrm{C}$ & $7.93 \pm 0.02^{\mathrm{i}}$ & $6.85 \pm 0.21^{\mathrm{egf}}$ & $76.53 \pm 0.43^{\mathrm{dc}}$ & $8.43 \pm 0.05^{\mathrm{ed}}$ & $8.19 \pm 0.26^{\mathrm{f}}$ & $414.73 \pm 1.03^{\text {ef }}$ \\
\hline & & $\mathrm{Cr}$ & $7.72 \pm 0.00^{\mathrm{j}}$ & $7.03 \pm 0.26^{\text {edf }}$ & $75.21 \pm 0.19^{\text {ih }}$ & $8.75 \pm 0.11^{\mathrm{d}}$ & $9.01 \pm 0.22^{\text {cde }}$ & $415.63 \pm 1.57^{\text {edf }}$ \\
\hline & \multirow{3}{*}{ Ginger } & $\mathrm{T}$ & $7.73 \pm 0.01^{j}$ & $6.10 \pm 0.08^{\mathrm{ihj}}$ & $77.39 \pm 0.02^{\mathrm{b}}$ & $7.00 \pm 0.04^{j}$ & $9.51 \pm 0.02^{\mathrm{cb}}$ & $410.58 \pm 0.50^{\mathrm{hg}}$ \\
\hline & & $\mathrm{C}$ & $7.93 \pm 0.01^{\mathrm{i}}$ & $7.53 \pm 0.30^{\mathrm{bdc}}$ & $75.99 \pm 0.17^{\mathrm{dfe}}$ & $7.54 \pm 0.01^{\text {ih }}$ & $8.94 \pm 0.12^{\text {de }}$ & $407.56 \pm 1.24^{\mathrm{hi}}$ \\
\hline & & $\mathrm{Cr}$ & $7.33 \pm 0.01^{\mathrm{k}}$ & $7.64 \pm 0.28^{\mathrm{bac}}$ & $74.21 \pm 0.33^{\mathrm{j}}$ & $8.15 \pm 0.10^{\mathrm{efg}}$ & $10.00 \pm 0.07^{\mathrm{b}}$ & $410.21 \pm 1.39^{\text {hg }}$ \\
\hline $\mathrm{CV}$ & & & 0.25 & 5.14 & 0.49 & 2.41 & 3.82 & 0.51 \\
\hline LSD & & & 0.04 & 0.59 & 0.61 & 0.34 & 0.55 & 3.47 \\
\hline
\end{tabular}

Where, TR=Treatment, LSD=list significant difference, $\mathrm{CV}=$ coefficient of variation; $\mathrm{MC}=\mathrm{moisture}$ content, $\mathrm{MD}=\mathrm{method}$ of drying, Spp.=species, $\mathrm{T}=$ tilapia, $\mathrm{C}=$ cat fish, $\mathrm{Cr}=$ common carp and the values are mean $\pm \mathrm{SE}$ in that the mean values followed by the same letter in a column are not significantly different at $5 \%$ level of significance.

Table 10 Proximate composition of dried fillets after the storage of two months

\begin{tabular}{|c|c|c|c|c|c|c|c|c|}
\hline MD & TR & Spp. & $\mathrm{MC}(\%)$ & $\operatorname{Ash}(\% \mathrm{db})$ & Protein $(\% \mathrm{db})$ & Fat $(\% \mathrm{db})$ & Total $\operatorname{carbs}(\% \mathrm{db})$ & Gross energy $(\mathrm{kcal} / 100 \mathrm{~g})$ \\
\hline \multirow{9}{*}{ Sun drying } & \multirow{3}{*}{ Control } & $\mathrm{T}$ & $12.21 \pm 0.02^{\mathrm{a}}$ & $6.32 \pm 0.28^{\text {ihg }}$ & $76.33 \pm 0.11^{\text {ed }}$ & $9.58 \pm 0.03^{\mathrm{bc}}$ & $7.77 \pm 0.33^{f}$ & $422.62 \pm 1.11^{\mathrm{a}}$ \\
\hline & & $\mathrm{C}$ & $11.73 \pm 0.01^{\mathrm{b}}$ & $7.95 \pm 0.12^{\mathrm{ba}}$ & $75.86 \pm 0.07^{\mathrm{fe}}$ & $9.46 \pm 0.30^{c}$ & $6.73 \pm 0.11^{\mathrm{h}}$ & $415.49 \pm 1.98^{\mathrm{dc}}$ \\
\hline & & $\mathrm{Cr}$ & $9.92 \pm 0.02^{\mathrm{c}}$ & $7.21 \pm 0.06^{\text {edc }}$ & $73.93 \pm 0.08^{\mathrm{ji}}$ & $10.03 \pm 0.09^{\mathrm{a}}$ & $8.83 \pm 0.00^{\mathrm{de}}$ & $421.33 \pm 0.57^{\mathrm{a}}$ \\
\hline & \multirow{3}{*}{ Garlic } & $\mathrm{T}$ & $9.13 \pm 0.00^{\mathrm{g}}$ & $5.90 \pm 0.19^{\mathrm{ij}}$ & $76.61 \pm 0.10^{\mathrm{cd}}$ & $9.51 \pm 0.01^{\mathrm{bc}}$ & $7.98 \pm 0.24^{\mathrm{f}}$ & $423.97 \pm 0.81^{\mathrm{a}}$ \\
\hline & & $\mathrm{C}$ & $9.61 \pm 0.03^{\mathrm{e}}$ & $6.98 \pm 0.07^{\text {edf }}$ & $75.64 \pm 0.10^{\mathrm{fg}}$ & $9.83 \pm 0.06^{\text {ba }}$ & $7.55 \pm 0.12^{\mathrm{gf}}$ & $421.27 \pm 0.59^{\mathrm{ba}}$ \\
\hline & & $\mathrm{Cr}$ & $8.33 \pm 0.01^{\mathrm{j}}$ & $8.20 \pm 0.19^{\mathrm{a}}$ & $73.07 \pm 0.25^{\mathrm{k}}$ & $9.83 \pm 0.06^{\mathrm{ba}}$ & $8.90 \pm 0.17^{\mathrm{de}}$ & $416.36 \pm 0.64^{\mathrm{c}}$ \\
\hline & \multirow{3}{*}{ Ginger } & $\mathrm{T}$ & $9.50 \pm 0.01^{\mathrm{f}}$ & $5.83 \pm 0.16^{\mathrm{ij}}$ & $78.89 \pm 0.10^{\mathrm{a}}$ & $8.23 \pm 0.21^{\text {ef }}$ & $7.05 \pm 0.24^{\mathrm{gh}}$ & $417.80 \pm 1.56^{\mathrm{bc}}$ \\
\hline & & $\mathrm{C}$ & $9.69 \pm 0.01^{\mathrm{d}}$ & $5.40 \pm 0.18^{\mathrm{j}}$ & $76.85 \pm 0.21^{\mathrm{cbd}}$ & $8.59 \pm 0.08^{\mathrm{d}}$ & $9.16 \pm 0.03^{\text {cde }}$ & $421.35 \pm 0.67^{\mathrm{a}}$ \\
\hline & & $\mathrm{Cr}$ & $8.85 \pm 0.01^{\mathrm{h}}$ & $7.96 \pm 0.17^{\text {ba }}$ & $73.46 \pm 0.11^{\mathrm{jk}}$ & $9.71 \pm 0.09^{\text {bac }}$ & $8.87 \pm 0.04^{\mathrm{de}}$ & $416.71 \pm 1.02^{c}$ \\
\hline \multirow{9}{*}{ Oven drying } & \multirow{3}{*}{ Control } & $\mathrm{T}$ & $8.11 \pm 0.01^{\mathrm{k}}$ & $6.56 \pm 0.10^{\mathrm{hgf}}$ & $75.92 \pm 0.04^{\mathrm{fe}}$ & $6.32 \pm 0.07^{\mathrm{k}}$ & $11.20 \pm 0.12^{\mathrm{a}}$ & $405.38 \pm 0.67^{\mathrm{g}}$ \\
\hline & & $\mathrm{C}$ & $8.62 \pm 0.01^{\mathrm{i}}$ & $6.80 \pm 0.34^{\mathrm{egf}}$ & $76.95 \pm 0.36^{\mathrm{cb}}$ & $7.31 \pm 0.07^{\mathrm{ij}}$ & $8.94 \pm 0.08^{\text {cde }}$ & $409.37 \pm 1.59^{\mathrm{fe}}$ \\
\hline & & $\mathrm{Cr}$ & $7.75 \pm 0.02^{1}$ & $7.68 \pm 0.25^{\mathrm{bac}}$ & $74.89 \pm 0.37^{\mathrm{h}}$ & $8.05 \pm 0.10^{\mathrm{fg}}$ & $9.38 \pm 0.48^{\mathrm{cd}}$ & $409.54 \pm 1.42^{\mathrm{fe}}$ \\
\hline & \multirow{3}{*}{ Garlic } & $\mathrm{T}$ & $8.88 \pm 0.00^{h}$ & $6.62 \pm 0.21^{\text {ehgf }}$ & $76.77 \pm 0.12^{\mathrm{cd}}$ & $7.85 \pm 0.21^{\mathrm{hg}}$ & $8.76 \pm 0.05^{\mathrm{e}}$ & $412.78 \pm 1.90^{\mathrm{de}}$ \\
\hline & & $\mathrm{C}$ & $8.60 \pm 0.02^{\mathrm{i}}$ & $6.73 \pm 0.21^{\mathrm{egf}}$ & $76.80 \pm 0.43^{\mathrm{cd}}$ & $8.43 \pm 0.05^{\mathrm{ed}}$ & $8.04 \pm 0.26^{\mathrm{f}}$ & $415.22 \pm 1.04^{\mathrm{dc}}$ \\
\hline & & $\mathrm{Cr}$ & $8.13 \pm 0.00^{\mathrm{k}}$ & $7.04 \pm 0.26^{\text {edf }}$ & $75.20 \pm 0.19^{\mathrm{hg}}$ & $8.74 \pm 0.11^{\mathrm{d}}$ & $9.02 \pm 0.22^{\text {cde }}$ & $415.52 \pm 1.57^{\mathrm{dc}}$ \\
\hline & \multirow{3}{*}{ Ginger } & $\mathrm{T}$ & $8.14 \pm 0.01^{\mathrm{k}}$ & $6.08 \pm 0.08^{\text {ih }}$ & $77.46 \pm 0.02^{b}$ & $6.97 \pm 0.04^{j}$ & $9.49 \pm 0.02^{\mathrm{cb}}$ & $410.57 \pm 0.50^{\mathrm{fe}}$ \\
\hline & & $\mathrm{C}$ & $8.60 \pm 0.01^{\mathrm{i}}$ & $7.41 \pm 0.31^{\mathrm{bdc}}$ & $76.25 \pm 0.18^{\text {fed }}$ & $7.53 \pm 0.01^{\text {ih }}$ & $8.81 \pm 0.12^{\mathrm{e}}$ & $408.01 \pm 1.25^{\mathrm{fg}}$ \\
\hline & & $\mathrm{Cr}$ & $7.74 \pm 0.01^{1}$ & $7.65 \pm 0.29^{\mathrm{bac}}$ & $74.20 \pm 0.34^{\mathrm{i}}$ & $8.14 \pm 0.10^{\text {efg }}$ & $10.01 \pm 0.07^{\mathrm{b}}$ & $410.08 \pm 1.39^{\mathrm{fe}}$ \\
\hline $\mathrm{CV}$ & & & 0.24 & 5.23 & 0.49 & 2.42 & 3.85 & 0.51 \\
\hline LSD & & & 0.04 & 0.60 & 0.61 & 0.34 & 0.55 & 3.49 \\
\hline
\end{tabular}

Where, $\mathrm{TR}=$ Treatment, $\mathrm{LSD}=$ list significant difference, $\mathrm{CV}=$ coefficient of variation; $\mathrm{MC}=$ moisture content, $\mathrm{MD}=\mathrm{method}$ of drying, $\mathrm{Spp} .=\mathrm{spec}$ ies, $\mathrm{T}=$ tilapia, $\mathrm{C}=$ cat fish, $\mathrm{Cr}=$ common carp and the values are mean $\pm \mathrm{SE}$ in that the mean values followed by the same letter in a column are not significantly different at $5 \%$ level of significance. 
Proximate Composition Dried Fish after the Storage of Two Months

All values of moisture content, ash, protein, fat, carbohydrate and gross energy were descended in their order due to the extended duration of storage period to two months of the fillets (Shina et al 2013) in their wet weight basis. The result of proximate compositions of dried fillets that differently treated and stored under ambient condition for two months is presented in Table 10.

The total moisture of the stored dried fish fillets was observed within the range of $7.7412 .21 \%$.The highest moisture contents were observed in untreated sundried fillets of the three species namely tilapia cat fish and carp with $12.21,11.73$ and $9.92 \%$ respectively. These values are statistically $(\mathrm{P}<0.05)$ different each other.

Proximate Composition of Dried Fish after the Storage of Three Months

Proximate compositions of differently treated and untreated dried fillets of tilapia / koroso /oreto, cat fish / ambassa / aggula and carp / duba/wit after three months storage were given in Table 11. The variation in proximate composition of the experimental fish fillets was not only because of species difference but might be due to difference in formulation and compositional difference between the garlic and ginger used. Moreover, proximate composition of the differently treated dried fillets stored for three months were presented in Table 11.

Significant $(\mathrm{P}<0.05)$ differences were observed in cat fish species in ash contents between untreated sun dried cat fish $(7.98 \%)$ and all the treated fillets except ginger treated oven dried cat fish $(7.43 \%)$ fillets. Significant $(\mathrm{P}<0.05)$ variation was observed in ash content between the untreated and garlic and ginger treated sundried fillets of carp with $7.23,8.23$ and $7.98 \%$ respectively. In general the amount of ash content of the three months stored fillets was laid within 5.41-8.23 percent.
Sensory Acceptability of Fresh and Dried Fillets before Storage

Sensory evaluation of the fresh fillets of the three fish species resulted in scores between 6 (like moderately) and 7 (like very much) in a hedonic scale of 7 points, for all the sensory parameters over the dried fillets (Table 12). Quality is a function of freshness; freshness is essential for quality but is not by itself a quality factor based on these sensorial analyses (Zambuchini, 2008).

Significant $(\mathrm{P}<0.05)$ differences have been noticed among the fresh fillets of the three species in all the parameters considered. Fresh cat fish exhibited the highest score (6.36) in color whereas carp and tilapia had the same score (6.18). All the dried fillets of the three fish showed the lowest scores with no statistical difference among them.

\section{Effect of Treatment Combination on Sensory Score of Dry Fillets}

Sensory scores of freshly dried fillets on color, odor, taste, texture and overall acceptability before storage are presented in Table 13. The scores on color varied from 4.98 of sundried fillets of carp fish not subjected to preservative up to 6.04 obtained by oven dried fillets of tilapia one of which was ginger treated and the other not treated. All these scores remained between like lightly and light moderately.

There were significant $(\mathrm{P}<0.05)$ differences between the untreated sundried fillets of all fish species and treated fillets for color, odor, taste and overall acceptability of dried fillets.

The dried fillets after the one month storage were evaluated by panelists get the values within 4 (Neither like nor dislike) to 6 (like moderately) hedonic values. Consumers noted significant $(\mathrm{P}<0.05)$ differences in color, odor, taste, texture and overall acceptability between the untreated sundried and treated fillets of fish.

Table 11 Proximate composition of dried fillets after the storage of three months

\begin{tabular}{|c|c|c|c|c|c|c|c|c|}
\hline MD & TR & Spp. & $\mathrm{MC}(\%)$ & $\operatorname{Ash}(\% \mathrm{db})$ & Protein $(\% \mathrm{db})$ & Fat $(\% \mathrm{db})$ & Total carbs $(\% \mathrm{db})$ & Gross energy $(\mathrm{kcal} / 100 \mathrm{~g})$ \\
\hline \multirow{9}{*}{ Sun drying } & & $\mathrm{T}$ & $13.12 \pm 0.02^{\mathrm{a}}$ & $6.30 \pm 0.28^{\text {hgi }}$ & $76.30 \pm 0.11^{\mathrm{ef}}$ & $9.66 \pm 0.03^{\text {bac }}$ & $7.74 \pm 0.33^{\mathrm{f}}$ & $423.12 \pm 1.13^{\mathrm{ba}}$ \\
\hline & Control & $\mathrm{C}$ & $12.30 \pm 0.01^{\mathrm{b}}$ & $7.98 \pm 0.12^{\mathrm{ba}}$ & $75.95 \pm 0.07^{\mathrm{f}}$ & $9.34 \pm 0.30^{\mathrm{c}}$ & $6.73 \pm 0.12^{\mathrm{h}}$ & $414.78 \pm 1.99^{\mathrm{ed}}$ \\
\hline & & $\mathrm{Cr}$ & $10.34 \pm 0.02^{\mathrm{d}}$ & $7.23 \pm 0.06^{\mathrm{edc}}$ & $73.93 \pm 0.08^{\mathrm{ji}}$ & $9.98 \pm 0.09^{\mathrm{a}}$ & $8.86 \pm 0.00^{\mathrm{de}}$ & $420.97 \pm 0.58^{\text {bac }}$ \\
\hline & & $\mathrm{T}$ & $10.03 \pm 0.00^{g}$ & $5.87 \pm 0.19^{\mathrm{ji}}$ & $76.59 \pm 0.11^{\text {ced }}$ & $9.59 \pm 0.01^{\mathrm{bc}}$ & $7.95 \pm 0.24^{\mathrm{f}}$ & $424.47 \pm 0.82^{\mathrm{a}}$ \\
\hline & Garlic & $\mathrm{C}$ & $10.18 \pm 0.03^{\mathrm{f}}$ & $6.99 \pm 0.07^{\text {edf }}$ & $75.73 \pm 0.10^{\mathrm{gf}}$ & $9.72 \pm 0.06^{\mathrm{ba}}$ & $7.56 \pm 0.12^{\mathrm{gf}}$ & $420.61 \pm 0.59^{\mathrm{bc}}$ \\
\hline & & $\mathrm{Cr}$ & $8.76 \pm 0.01^{1}$ & $8.23 \pm 0.19^{\mathrm{a}}$ & $73.07 \pm 0.25^{\mathrm{k}}$ & $9.78 \pm 0.06^{\mathrm{ba}}$ & $8.92 \pm 0.17^{\mathrm{de}}$ & $415.98 \pm 0.64^{\text {ed }}$ \\
\hline & & $\mathrm{T}$ & $10.40 \pm 0.01^{\mathrm{c}}$ & $5.80 \pm 0.16^{\mathrm{ji}}$ & $78.88 \pm 0.10^{\mathrm{a}}$ & $8.29 \pm 0.21^{\mathrm{ef}}$ & $7.03 \pm 0.24^{\mathrm{gh}}$ & $418.24 \pm 1.57^{\mathrm{dc}}$ \\
\hline & Ginger & $\mathrm{C}$ & $10.26 \pm 0.01^{\mathrm{e}}$ & $5.41 \pm 0.18^{\mathrm{j}}$ & $76.95 \pm 0.21^{\mathrm{cbd}}$ & $8.47 \pm 0.08^{\mathrm{ed}}$ & $9.17 \pm 0.03^{\mathrm{dce}}$ & $420.70 \pm 0.68^{\mathrm{bc}}$ \\
\hline & & $\mathrm{Cr}$ & $9.27 \pm 0.01^{\mathrm{i}}$ & $7.98 \pm 0.17^{\text {ba }}$ & $73.46 \pm 0.11^{\mathrm{jk}}$ & $9.65 \pm 0.09^{\mathrm{bac}}$ & $8.91 \pm 0.04^{\mathrm{de}}$ & $416.34 \pm 1.03^{\mathrm{ed}}$ \\
\hline \multirow{9}{*}{ Oven drying } & & $\mathrm{T}$ & $9.01 \pm 0.01^{\mathrm{k}}$ & $6.54 \pm 0.10^{\text {hgf }}$ & $75.89 \pm 0.04^{\mathrm{f}}$ & $6.37 \pm 0.07^{j}$ & $11.20 \pm 0.12^{\mathrm{a}}$ & $405.68 \pm 0.67^{1}$ \\
\hline & Control & $\mathrm{C}$ & $9.19 \pm 0.01^{\mathrm{j}}$ & $6.81 \pm 0.34^{\text {egf }}$ & $77.04 \pm 0.36^{\mathrm{cb}}$ & $7.18 \pm 0.07^{\text {ih }}$ & $8.97 \pm 0.08^{\mathrm{dce}}$ & $408.65 \pm 1.60^{\text {gih }}$ \\
\hline & & $\mathrm{Cr}$ & $8.18 \pm 0.02^{\mathrm{n}}$ & $7.70 \pm 0.25^{\mathrm{bac}}$ & $74.90 \pm 0.38^{\mathrm{h}}$ & $7.99 \pm 0.10^{\mathrm{gf}}$ & $9.41 \pm 0.48^{\mathrm{dc}}$ & $409.13 \pm 1.42^{\text {gih }}$ \\
\hline & & $\mathrm{T}$ & $9.79 \pm 0.00^{\mathrm{h}}$ & $6.60 \pm 0.22^{\text {hgf }}$ & $76.75 \pm 0.13^{\mathrm{ced}}$ & $7.91 \pm 0.22^{g}$ & $8.74 \pm 0.05^{\mathrm{e}}$ & $413.16 \pm 1.92^{\mathrm{ef}}$ \\
\hline & Garlic & $\mathrm{C}$ & $9.17 \pm 0.02^{\mathrm{j}}$ & $6.74 \pm 0.21^{\mathrm{egf}}$ & $76.89 \pm 0.43^{\text {cebd }}$ & $8.30 \pm 0.05^{\mathrm{ef}}$ & $8.07 \pm 0.27^{\mathrm{f}}$ & $414.54 \pm 1.05^{\mathrm{e}}$ \\
\hline & & $\mathrm{Cr}$ & $8.55 \pm 0.00^{\mathrm{m}}$ & $7.06 \pm 0.26^{\mathrm{edf}}$ & $75.21 \pm 0.19^{\mathrm{gh}}$ & $8.68 \pm 0.11^{\mathrm{d}}$ & $9.05 \pm 0.22^{\mathrm{dce}}$ & $415.15 \pm 1.58^{\mathrm{ed}}$ \\
\hline & & $\mathrm{T}$ & $9.05 \pm 0.01^{\mathrm{k}}$ & $6.05 \pm 0.08^{\text {hi }}$ & $77.45 \pm 0.02^{b}$ & $7.03 \pm 0.04^{i}$ & $9.47 \pm 0.02^{\mathrm{c}}$ & $410.93 \pm 0.50^{g f}$ \\
\hline & Ginger & $\mathrm{C}$ & $9.17 \pm 0.01^{\mathrm{j}}$ & $7.43 \pm 0.31^{\mathrm{bdc}}$ & $76.34 \pm 0.18^{\mathrm{efd}}$ & $7.40 \pm 0.01^{\mathrm{h}}$ & $8.83 \pm 0.13^{\mathrm{e}}$ & $407.27 \pm 1.25^{\text {ih }}$ \\
\hline & & $\mathrm{Cr}$ & $8.16 \pm 0.01^{\mathrm{n}}$ & $7.68 \pm 0.29^{\mathrm{bac}}$ & $74.20 \pm 0.34^{\mathrm{i}}$ & $8.08 \pm 0.10^{\mathrm{gf}}$ & $10.04 \pm 0.07^{b}$ & $409.68 \pm 1.40^{\mathrm{gfh}}$ \\
\hline $\mathrm{CV}$ & & & 0.22 & 5.26 & 0.49 & 2.45 & 3.88 & 0.51 \\
\hline LSD & & & 0.04 & 0.60 & 0.62 & 0.35 & 0.56 & 3.52 \\
\hline
\end{tabular}

Where, TR=Treatment, LSD=list significant difference, $C V=$ coefficient of variation; $M C=$ moisture content, $\mathrm{MD}=$ method of drying, Spp.=species, $\mathrm{T}=$ tilapia, $\mathrm{C}=$ cat fish, $\mathrm{Cr}=$ common carp and the values are mean $\pm \mathrm{SE}$ in that the mean values followed by the same letter in a column are not different at $5 \%$ level of significance. 
Table 12 Sensory evaluation scores of fresh and dried fish

\begin{tabular}{lccccc}
\hline Spp. & Color & Odor & Taste & Texture & Over all acceptability \\
\hline Fresh cat fish & $6.36 \pm 0.07^{\mathrm{a}}$ & $6.38 \pm 0.07^{\mathrm{a}}$ & $6.18 \pm 0.06^{\mathrm{a}}$ & $6.30 \pm 0.07^{\mathrm{b}}$ & $6.04 \pm 0.03^{\mathrm{b}}$ \\
Fresh carp & $6.18 \pm 0.07^{\mathrm{b}}$ & $6.34 \pm 0.09^{\mathrm{ba}}$ & $6.02 \pm 0.03^{\mathrm{b}}$ & $6.78 \pm 0.07^{\mathrm{a}}$ & $6.86 \pm 0.06^{\mathrm{a}}$ \\
Fresh tilapia & $6.18 \pm 0.05^{\mathrm{b}}$ & $6.18 \pm 0.05^{\mathrm{b}}$ & $6.10 \pm 0.04^{\mathrm{ba}}$ & $6.30 \pm 0.07^{\mathrm{b}}$ & $6.78 \pm 0.06^{\mathrm{a}}$ \\
Dried cat fish & $5.71 \pm 0.04^{\mathrm{c}}$ & $5.75 \pm 0.04^{\mathrm{c}}$ & $5.81 \pm 0.03^{\mathrm{c}}$ & $5.46 \pm 0.05^{\mathrm{c}}$ & $5.86 \pm 0.03^{\mathrm{cb}}$ \\
Dried carp & $5.68 \pm 0.04^{\mathrm{c}}$ & $5.80 \pm 0.03^{\mathrm{c}}$ & $5.62 \pm 0.04^{\mathrm{d}}$ & $5.28 \pm 0.04^{\mathrm{c}}$ & $5.72 \pm 0.03^{\mathrm{cd}}$ \\
Dried tilapia & $5.78 \pm 0.03^{\mathrm{c}}$ & $5.70 \pm 0.04^{\mathrm{c}}$ & $5.62 \pm 0.03^{\mathrm{d}}$ & $5.44 \pm 0.05^{\mathrm{c}}$ & $5.94 \pm 0.04^{\mathrm{d}}$ \\
\hline CV & 10.35 & 10.47 & 10.22 & 13.40 & 10.00 \\
LSD & 0.162 & 0.165 & 0.159 & 0.197 & 1.740 \\
\hline
\end{tabular}

Where, Spp. $=$ species, $\mathrm{CV}=$ coefficient of variances, $\mathrm{LSD}=$ least significant differences and the values are mean $\pm \mathrm{SE}$ in that the mean values followed by the same letter in a column are not significantly different at $5 \%$ level of significance.

Table 13 Effect of treatment combination on sensory score dry fillets before storage

\begin{tabular}{|c|c|c|c|c|c|c|c|}
\hline Drying method & Treatments & Spp & Color & Odor & Taste & Texture & Overall acceptability \\
\hline \multirow{9}{*}{ Sun drying } & \multirow{3}{*}{ Control } & $\mathrm{T}$ & $5.00 \pm 0.03^{\mathrm{f}}$ & $4.76 \pm 0.13^{\mathrm{d}}$ & $5.02 \pm 0.05^{\mathrm{f}}$ & $5.00 \pm 0.06^{\text {gef }}$ & $4.84 \pm 0.10^{\mathrm{e}}$ \\
\hline & & $\mathrm{C}$ & $4.98 \pm 0.08^{\mathrm{f}}$ & $4.96 \pm 0.09^{c}$ & $4.86 \pm 0.12^{\mathrm{gf}}$ & $4.92 \pm 0.07^{\mathrm{g}}$ & $5.04 \pm 0.09^{\mathrm{d}}$ \\
\hline & & $\mathrm{Cr}$ & $5.04 \pm 0.06^{\mathrm{f}}$ & $5.08 \pm 0.09^{c}$ & $4.78 \pm 0.12^{\mathrm{g}}$ & $4.98 \pm 0.08^{\mathrm{gf}}$ & $4.88 \pm 0.09^{\mathrm{e}}$ \\
\hline & \multirow{3}{*}{ Garlic } & $\mathrm{T}$ & $5.98 \pm 0.02^{\mathrm{ba}}$ & $6.00 \pm 0.00^{\mathrm{a}}$ & $5.30 \pm 0.08^{\mathrm{e}}$ & $5.18 \pm 0.12^{\text {gcefd }}$ & $5.62 \pm 0.09^{c}$ \\
\hline & & $\mathrm{C}$ & $6.02 \pm 0.02^{\text {ba }}$ & $6.02 \pm 0.02^{\mathrm{a}}$ & $6.00 \pm 0.00^{\mathrm{a}}$ & $6.20 \pm 0.06^{\mathrm{cefd}}$ & $6.00 \pm 0.00^{\mathrm{b}}$ \\
\hline & & $\mathrm{Cr}$ & $5.44 \pm 0.08^{\mathrm{e}}$ & $6.02 \pm 0.02^{\mathrm{a}}$ & $6.00 \pm 0.00^{\mathrm{a}}$ & $5.12 \pm 0.13^{\text {gefd }}$ & $6.00 \pm 0.00^{\mathrm{b}}$ \\
\hline & \multirow{3}{*}{ Ginger } & $\mathrm{T}$ & $5.60 \pm 0.10^{\text {ed }}$ & $6.02 \pm 0.02^{\mathrm{a}}$ & $5.30 \pm 0.11^{\text {ed }}$ & $5.32 \pm 0.11^{\mathrm{cbd}}$ & $6.02 \pm 0.02^{b}$ \\
\hline & & $\mathrm{C}$ & $5.50 \pm 0.08^{\mathrm{e}}$ & $5.74 \pm 0.10^{b}$ & $6.00 \pm 0.00^{\mathrm{a}}$ & $6.52 \pm 0.09^{\mathrm{a}}$ & $6.00 \pm 0.00^{\mathrm{b}}$ \\
\hline & & $\mathrm{Cr}$ & $6.00 \pm 0.00^{\text {ba }}$ & $6.00 \pm 0.00^{\mathrm{a}}$ & $5.50 \pm 0.11^{\mathrm{cd}}$ & $5.26 \pm 0.07^{\text {cebd }}$ & $5.62 \pm 0.07^{\mathrm{c}}$ \\
\hline \multirow{9}{*}{ Oven drying } & \multirow{3}{*}{ Control } & $\mathrm{T}$ & $6.04 \pm 0.03^{\mathrm{a}}$ & $5.70 \pm 0.10^{b}$ & $6.00 \pm 0.00^{\mathrm{a}}$ & $5.42 \pm 0.09^{\mathrm{cb}}$ & $6.06 \pm 0.03^{b}$ \\
\hline & & $\mathrm{C}$ & $5.86 \pm 0.10^{\mathrm{ba}}$ & $5.76 \pm 0.11^{\mathrm{b}}$ & $6.02 \pm 0.02^{\mathrm{a}}$ & $5.44 \pm 0.08^{\mathrm{cb}}$ & $6.00 \pm 0.00^{\mathrm{b}}$ \\
\hline & & $\mathrm{Cr}$ & $6.02 \pm 0.02^{\text {ba }}$ & $6.00 \pm 0.00^{\mathrm{a}}$ & $5.78 \pm 0.08^{b}$ & $5.44 \pm 0.11^{\mathrm{cb}}$ & $5.78 \pm 0.07^{\mathrm{c}}$ \\
\hline & \multirow{3}{*}{ Garlic } & $\mathrm{T}$ & $6.02 \pm 0.02^{\mathrm{ba}}$ & $6.02 \pm 0.02^{\mathrm{a}}$ & $6.00 \pm 0.00^{\mathrm{a}}$ & $5.26 \pm 0.08^{\text {cebd }}$ & $6.96 \pm 0.03^{\mathrm{a}}$ \\
\hline & & $\mathrm{C}$ & $5.90 \pm 0.11^{\text {bac }}$ & $6.02 \pm 0.02^{\mathrm{a}}$ & $6.00 \pm 0.00^{\mathrm{a}}$ & $5.34 \pm 0.13^{\mathrm{cbd}}$ & $6.04 \pm 0.03^{\mathrm{b}}$ \\
\hline & & $\mathrm{Cr}$ & $5.76 \pm 0.11^{\mathrm{dc}}$ & $6.04 \pm 0.03^{\mathrm{a}}$ & $5.66 \pm 0.10^{\mathrm{cb}}$ & $5.38 \pm 0.11^{\mathrm{cbd}}$ & $6.00 \pm 0.00^{\mathrm{b}}$ \\
\hline & \multirow{3}{*}{ Ginger } & $\mathrm{T}$ & $6.04 \pm 0.10^{\mathrm{a}}$ & $5.70 \pm 0.10^{b}$ & $6.02 \pm 0.02^{\mathrm{a}}$ & $6.48 \pm 0.09^{\mathrm{a}}$ & $6.14 \pm 0.06^{b}$ \\
\hline & & $\mathrm{C}$ & $6.02 \pm 0.02^{\text {ba }}$ & $6.00 \pm 0.00^{\mathrm{b}}$ & $6.00 \pm 0.00^{\mathrm{a}}$ & $5.36 \pm 0.09^{\mathrm{cbd}}$ & $6.08 \pm 0.04^{b}$ \\
\hline & & $\mathrm{Cr}$ & $5.84 \pm 0.10^{\mathrm{bc}}$ & $5.68 \pm 0.10^{b}$ & $6.00 \pm 0.00^{\mathrm{a}}$ & $5.50 \pm 0.08^{\mathrm{b}}$ & $6.02 \pm 0.08^{b}$ \\
\hline$\overline{\mathrm{CV}}$ & & & 8.78 & 8.57 & 8.10 & 12.33 & 6.84 \\
\hline LSD & & & 0.20 & 0.19 & 0.18 & 0.26 & 1.96 \\
\hline
\end{tabular}

Where, $\mathrm{LSD}=$ list significant difference, $\mathrm{CV}=$ coefficient of variation, $\mathrm{Spp} .=$ species, $\mathrm{T}=$ tilapia, $\mathrm{C}=\mathrm{cat}$ fish, $\mathrm{Cr}=\mathrm{common}$ carp and the values are mean $\pm \mathrm{SE}$ in that the mean values followed by the same letter in a column are not significantly different at $5 \%$ level of significance.

Table 14 Sensory acceptability test results of dried fish fillets stored for one month

\begin{tabular}{|c|c|c|c|c|c|c|c|}
\hline Drying method & Treatments & Spp. & Color & Odor & Taste & Texture & Overall acceptability \\
\hline \multirow{9}{*}{ Sun drying } & \multirow{3}{*}{ Control } & $\mathrm{T}$ & $4.54 \pm 0.10^{f}$ & $4.56 \pm 0.09^{\mathrm{e}}$ & $4.48 \pm 0.09^{j}$ & $4.58 \pm 0.08^{f}$ & $4.62 \pm 0.09^{\mathrm{g}}$ \\
\hline & & $\mathrm{C}$ & $4.58 \pm 0.10^{f}$ & $4.06 \pm 0.03^{\mathrm{f}}$ & $4.56 \pm 0.10^{\mathrm{ij}}$ & $4.56 \pm 0.10^{f}$ & $4.08 \pm 0.04^{\mathrm{h}}$ \\
\hline & & $\mathrm{Cr}$ & $4.48 \pm 0.12^{\mathrm{f}}$ & $4.06 \pm 0.03^{\mathrm{f}}$ & $4.72 \pm 0.08^{\text {ih }}$ & $4.06 \pm 0.03^{\mathrm{g}}$ & $4.06 \pm 0.03^{\mathrm{h}}$ \\
\hline & \multirow{3}{*}{ Garlic } & $\mathrm{T}$ & $5.98 \pm 0.02^{\mathrm{a}}$ & $5.04 \pm 0.03^{\mathrm{d}}$ & $5.04 \pm 0.03^{\text {gfe }}$ & $5.12 \pm 0.05^{\mathrm{de}}$ & $5.08 \pm 0.04^{\mathrm{fe}}$ \\
\hline & & $\mathrm{C}$ & $5.04 \pm 0.03^{\mathrm{e}}$ & $5.04 \pm 0.03^{\mathrm{d}}$ & $6.00 \pm 0.00^{\mathrm{a}}$ & $5.08 \pm 0.04^{\mathrm{e}}$ & $5.06 \pm 0.03^{\mathrm{f}}$ \\
\hline & & $\mathrm{Cr}$ & $6.00 \pm 0.00^{\mathrm{a}}$ & $5.04 \pm 0.03^{\mathrm{d}}$ & $5.04 \pm 0.03^{\mathrm{gfe}}$ & $5.04 \pm 0.03^{\mathrm{e}}$ & $5.04 \pm 0.03^{\mathrm{f}}$ \\
\hline & \multirow{3}{*}{ Ginger } & $\mathrm{T}$ & $5.08 \pm 0.05^{\mathrm{e}}$ & $6.00 \pm 0.00^{\mathrm{a}}$ & $5.04 \pm 0.03^{\mathrm{gfe}}$ & $6.00 \pm 0.00^{\mathrm{a}}$ & $5.46 \pm 0.07^{\mathrm{a}}$ \\
\hline & & $\mathrm{C}$ & $5.02 \pm 0.02^{\mathrm{e}}$ & $6.00 \pm 0.00^{\mathrm{a}}$ & $4.88 \pm 0.10^{\mathrm{gh}}$ & $6.00 \pm 0.00^{\mathrm{a}}$ & $5.22 \pm 0.06^{\text {becd }}$ \\
\hline & & $\mathrm{Cr}$ & $5.04 \pm 0.03^{\mathrm{e}}$ & $6.00 \pm 0.00^{\mathrm{a}}$ & $4.90 \pm 0.10^{\mathrm{gf}}$ & $6.00 \pm 0.00^{\mathrm{a}}$ & $5.24 \pm 0.06^{\text {bcd }}$ \\
\hline \multirow{9}{*}{ Oven drying } & \multirow{3}{*}{ Control } & $\mathrm{T}$ & $5.26 \pm 0.06^{\mathrm{d}}$ & $5.08 \pm 0.04^{\mathrm{d}}$ & $5.04 \pm 0.09^{\mathrm{gfe}}$ & $5.30 \pm 0.07^{c}$ & $5.30 \pm 0.07^{\mathrm{bc}}$ \\
\hline & & $\mathrm{C}$ & $5.04 \pm 0.03^{\mathrm{e}}$ & $5.34 \pm 0.07^{\mathrm{c}}$ & $5.08 \pm 0.04^{\mathrm{dce}}$ & $5.08 \pm 0.04^{\mathrm{e}}$ & $5.14 \pm 0.05^{\text {fed }}$ \\
\hline & & $\mathrm{Cr}$ & $5.52 \pm 0.09^{c}$ & $5.06 \pm 0.04^{\mathrm{d}}$ & $5.22 \pm 0.06^{\mathrm{dc}}$ & $5.08 \pm 0.05^{\mathrm{e}}$ & $5.12 \pm 0.05^{\text {fed }}$ \\
\hline & \multirow{3}{*}{ Garlic } & $\mathrm{T}$ & $5.74 \pm 0.06^{b}$ & $5.04 \pm 0.03^{\mathrm{d}}$ & $5.06 \pm 0.03^{\mathrm{dfe}}$ & $5.32 \pm 0.08^{c}$ & $5.32 \pm 0.07^{\mathrm{ba}}$ \\
\hline & & $\mathrm{C}$ & $5.02 \pm 0.02^{\mathrm{e}}$ & $5.22 \pm 0.06^{\mathrm{c}}$ & $5.64 \pm 0.07^{\mathrm{b}}$ & $5.04 \pm 0.03^{\mathrm{e}}$ & $5.06 \pm 0.03^{f}$ \\
\hline & & $\mathrm{Cr}$ & $5.54 \pm 0.07^{\mathrm{c}}$ & $5.04 \pm 0.03^{\mathrm{d}}$ & $5.02 \pm 0.02^{\mathrm{gfe}}$ & $5.26 \pm 0.06^{\mathrm{dc}}$ & $5.04 \pm 0.03^{\mathrm{f}}$ \\
\hline & \multirow{3}{*}{ Ginger } & $\mathrm{T}$ & $5.26 \pm 0.06^{\mathrm{d}}$ & $5.50 \pm 0.07^{b}$ & $5.02 \pm 0.02^{\text {gfe }}$ & $5.52 \pm 0.07^{b}$ & $5.26 \pm 0.06^{\mathrm{bcd}}$ \\
\hline & & $\mathrm{C}$ & $5.02 \pm 0.02^{\mathrm{e}}$ & $5.52 \pm 0.07^{\mathrm{b}}$ & $5.24 \pm 0.06^{\mathrm{c}}$ & $5.50 \pm 0.07^{\mathrm{b}}$ & $5.16 \pm 0.05^{\mathrm{fecd}}$ \\
\hline & & $\mathrm{Cr}$ & $5.04 \pm 0.03^{\mathrm{e}}$ & $5.50 \pm 0.07^{b}$ & $5.24 \pm 0.06^{c}$ & $5.56 \pm 0.07^{\mathrm{b}}$ & $5.22 \pm 0.06^{\text {becd }}$ \\
\hline $\mathrm{CV}$ & & & 8.07 & 6.55 & 8.84 & 7.51 & 7.49 \\
\hline LSD & & & 0.16 & 0.13 & 0.18 & 0.15 & 0.15 \\
\hline
\end{tabular}

Where, $\mathrm{LSD}=$ list significant difference, $\mathrm{CV}=$ coefficient of variation, Spp.=species, $\mathrm{T}=\mathrm{tilapia}, \mathrm{C}=\mathrm{cat}$ fish, $\mathrm{Cr}=\mathrm{common}$ carp and the values are mean $\pm \mathrm{SE}$ in that the mean values followed by the same letter in a column are not significantly different at $5 \%$ level of significance. 
Sensory Acceptability Test Results of Dried Fish Fillets after Two Months Storage

Most of the dried fillets after two months storage were evaluated by panelists get the values within 4 (Neither like nor dislike) to 5 (like slightly) hedonic values. No differences $(\mathrm{P}>0.05)$ were observed between untreated sundried fillets of three species for the individual attributes: color, odor, texture and overall acceptability.

There were steady decreased values in all sensorial parameters through the progressive storage periods of two months. Panelist found or preferred the flavor of the garlic and ginger to the allicinic effect and pungency flavor of the treated fish fillets respectively. Overall acceptability scores decreased as storage time increased to two months in all the fish fillets.
Sensory Acceptability Test Results of Dried Fish Fillets after three Months Storage

The dried fillets after the three months storage period were evaluated by panelists get the values within 2.92 to 5 means from the hedonic values. There was significant variation $(\mathrm{P}<0.05)$ in some of the tested parameters.

\section{Fish}

Effect of Storage Time on Nutritional Value of Dried

Oven drying and sun drying in this study could not affect the nutritional components of fillets. Statistical $(\mathrm{P}<0.05)$ differences were observed in these components based on method of drying except ash and crude protein content through the storage time.

Table 15 Sensory acceptability test results of dried fish fillets stored for two months

\begin{tabular}{|c|c|c|c|c|c|c|c|}
\hline Drying method & Treatment & Spp & Color & Odor & Taste & Texture & Overallacceptability \\
\hline \multirow{9}{*}{ Sun drying } & \multirow{3}{*}{ Control } & $\mathrm{T}$ & $4.00 \pm 0.00^{\mathrm{g}}$ & $4.00 \pm 0.00^{\mathrm{h}}$ & $3.86 \pm 0.05^{\mathrm{f}}$ & $4.00 \pm 0.03^{f}$ & $4.00 \pm 0.00^{\mathrm{e}}$ \\
\hline & & $\mathrm{C}$ & $4.00 \pm 0.00^{\mathrm{g}}$ & $4.00 \pm 0.04^{\mathrm{h}}$ & $4.00 \pm 0.00^{\mathrm{fe}}$ & $4.00 \pm 0.00^{\mathrm{f}}$ & $4.02 \pm 0.02^{\mathrm{e}}$ \\
\hline & & $\mathrm{Cr}$ & $4.00 \pm 0.03^{\mathrm{g}}$ & $3.98 \pm 0.03^{\mathrm{h}}$ & $4.08 \pm 0.04^{\mathrm{e}}$ & $4.00 \pm 0.00^{\mathrm{f}}$ & $4.00 \pm 0.00^{\mathrm{e}}$ \\
\hline & \multirow{3}{*}{ Garlic } & $\mathrm{T}$ & $5.00 \pm 0.00^{\mathrm{e}}$ & $5.00 \pm 0.00^{\text {ed }}$ & $4.38 \pm 0.07^{\mathrm{d}}$ & $5.00 \pm 0.00^{\mathrm{cd}}$ & $5.00 \pm 0.00^{b}$ \\
\hline & & $\mathrm{C}$ & $6.00 \pm 0.00^{\mathrm{a}}$ & $4.40 \pm 0.07^{\mathrm{f}}$ & $5.00 \pm 0.00^{\mathrm{b}}$ & $5.00 \pm 0.00^{\mathrm{cd}}$ & $5.00 \pm 0.00^{\mathrm{b}}$ \\
\hline & & $\mathrm{Cr}$ & $5.00 \pm 0.00^{\mathrm{e}}$ & $5.00 \pm 0.00^{\text {ed }}$ & $5.00 \pm 0.00^{\mathrm{b}}$ & $4.20 \pm 0.06^{\mathrm{e}}$ & $5.00 \pm 0.00^{\mathrm{b}}$ \\
\hline & \multirow{3}{*}{ Ginger } & $\mathrm{T}$ & $5.00 \pm 0.00^{\mathrm{e}}$ & $5.00 \pm 0.00^{\text {ed }}$ & $5.00 \pm 0.00^{\mathrm{b}}$ & $5.00 \pm 0.00^{\mathrm{cd}}$ & $5.00 \pm 0.00^{\mathrm{b}}$ \\
\hline & & $\mathrm{C}$ & $6.00 \pm 0.00^{\mathrm{a}}$ & $4.20 \pm 0.06^{\mathrm{g}}$ & $5.00 \pm 0.00^{\mathrm{b}}$ & $5.00 \pm 0.00^{\mathrm{cd}}$ & $5.00 \pm 0.00^{\mathrm{b}}$ \\
\hline & & $\mathrm{Cr}$ & $5.00 \pm 0.00^{\mathrm{e}}$ & $5.00 \pm 0.00^{\text {ed }}$ & $5.00 \pm 0.00^{\mathrm{b}}$ & $5.00 \pm 0.00^{\mathrm{cd}}$ & $5.00 \pm 0.00^{\mathrm{b}}$ \\
\hline \multirow{9}{*}{ Oven drying } & \multirow{3}{*}{ Control } & $\mathrm{T}$ & $5.20 \pm 0.06^{\mathrm{d}}$ & $5.00 \pm 0.00^{\text {ed }}$ & $4.50 \pm 0.18^{\mathrm{dc}}$ & $4.90 \pm 0.12^{\mathrm{d}}$ & $5.10 \pm 0.08^{b}$ \\
\hline & & $\mathrm{C}$ & $5.00 \pm 0.00^{\mathrm{e}}$ & $5.12 \pm 0.16^{\mathrm{bcd}}$ & $5.10 \pm 0.04^{\mathrm{b}}$ & $5.10 \pm 0.04^{\mathrm{cb}}$ & $4.72 \pm 0.09^{\mathrm{dc}}$ \\
\hline & & $\mathrm{Cr}$ & $5.40 \pm 0.13^{\mathrm{c}}$ & $4.40 \pm 0.09^{\mathrm{f}}$ & $5.10 \pm 0.04^{\mathrm{b}}$ & $4.90 \pm 0.12^{\mathrm{d}}$ & $4.80 \pm 0.11^{\mathrm{c}}$ \\
\hline & \multirow{3}{*}{ Garlic } & $\mathrm{T}$ & $5.70 \pm 0.09^{b}$ & $5.10 \pm 0.04^{\mathrm{cd}}$ & $5.10 \pm 0.04^{b}$ & $4.30 \pm 0.09^{\mathrm{e}}$ & $5.30 \pm 0.09^{\mathrm{a}}$ \\
\hline & & $\mathrm{C}$ & $5.00 \pm 0.00^{\mathrm{e}}$ & $5.20 \pm 0.06^{\mathrm{bc}}$ & $5.70 \pm 0.07^{\mathrm{a}}$ & $5.10 \pm 0.04^{\mathrm{cb}}$ & $5.10 \pm 0.04^{b}$ \\
\hline & & $\mathrm{Cr}$ & $5.50 \pm 0.07^{\mathrm{c}}$ & $5.10 \pm 0.04^{\text {cd }}$ & $5.10 \pm 0.04^{\mathrm{b}}$ & $5.20 \pm 0.06^{\mathrm{b}}$ & $5.10 \pm 0.04^{\mathrm{b}}$ \\
\hline & \multirow{3}{*}{ Ginger } & $\mathrm{T}$ & $4.40 \pm 0.11^{\mathrm{f}}$ & $5.30 \pm 0.07^{\text {ba }}$ & $5.10 \pm 0.04^{b}$ & $5.40 \pm 0.11^{\mathrm{a}}$ & $4.60 \pm 0.09^{d}$ \\
\hline & & $\mathrm{C}$ & $5.10 \pm 0.04^{\mathrm{ed}}$ & $5.40 \pm 0.07^{\mathrm{a}}$ & $4.60 \pm 0.16^{\mathrm{c}}$ & $5.00 \pm 0.11^{\mathrm{cd}}$ & $5.30 \pm 0.07^{\mathrm{a}}$ \\
\hline & & $\mathrm{Cr}$ & $5.10 \pm 0.04^{\mathrm{ed}}$ & $4.90 \pm 0.15^{\mathrm{e}}$ & $4.34 \pm 0.16^{\mathrm{d}}$ & $5.50 \pm 0.07^{\mathrm{a}}$ & $5.00 \pm 0.00^{\mathrm{b}}$ \\
\hline$\overline{\mathrm{CV}}$ & & & 7.53 & 10.11 & 11.33 & 9.67 & 7.80 \\
\hline LSD & & & 0.15 & 0.19 & 0.21 & 0.18 & 0.15 \\
\hline
\end{tabular}

Where, $\mathrm{LSD}=$ list significant difference, $\mathrm{CV}=$ coefficient of variation, $\mathrm{Spp} .=$ species, $\mathrm{T}=$ tilapia, $\mathrm{C}=$ cat fish, $\mathrm{Cr}=$ common carp and the values are mean $\pm \mathrm{SE}$ in that the mean values followed by the same letter in a column are not significantly different at $5 \%$ level of significance.

Table 16 Sensory acceptability test results of dried fish fillets stored for three months

\begin{tabular}{|c|c|c|c|c|c|c|}
\hline Drying method & Treatment & Spp. & Color & Odor & Texture & Overall acceptability \\
\hline \multirow{9}{*}{ Sun drying } & \multirow{3}{*}{ Control } & $\mathrm{T}$ & $3.20 \pm 0.21^{\mathrm{h}}$ & $3.72 \pm 0.14^{\mathrm{g}}$ & $3.84 \pm 0.13^{\mathrm{g}}$ & $3.60 \pm 0.11^{\mathrm{f}}$ \\
\hline & & $\mathrm{C}$ & $3.00 \pm 0.00^{\text {ih }}$ & $4.14 \pm 0.13^{f}$ & $4.08 \pm 0.13^{\mathrm{f}}$ & $3.66 \pm 0.11^{\mathrm{f}}$ \\
\hline & & $\mathrm{Cr}$ & $2.92 \pm 0.06^{\mathrm{i}}$ & $3.00 \pm 0.00^{\mathrm{h}}$ & $3.00 \pm 0.00^{\mathrm{h}}$ & $3.00 \pm 0.03^{\mathrm{g}}$ \\
\hline & \multirow{3}{*}{ Garlic } & $\mathrm{T}$ & $4.00 \pm 0.00^{\mathrm{f}}$ & $4.54 \pm 0.11^{\mathrm{ed}}$ & $4.96 \pm 0.03^{\mathrm{a}}$ & $4.92 \pm 0.04^{\mathrm{ba}}$ \\
\hline & & $\mathrm{C}$ & $4.34 \pm 0.08^{\text {ed }}$ & $4.60 \pm 0.07^{\mathrm{edc}}$ & $4.98 \pm 0.02^{\mathrm{a}}$ & $4.96 \pm 0.03^{\mathrm{ba}}$ \\
\hline & & $\mathrm{Cr}$ & $3.62 \pm 0.13^{g}$ & $4.98 \pm 0.02^{\mathrm{a}}$ & $4.98 \pm 0.11^{\mathrm{a}}$ & $4.98 \pm 0.02^{\mathrm{a}}$ \\
\hline & \multirow{3}{*}{ Ginger } & $\mathrm{T}$ & $4.98 \pm 0.02^{\mathrm{a}}$ & $4.98 \pm 0.02^{\mathrm{a}}$ & $4.30 \pm 0.03^{\mathrm{e}}$ & $4.98 \pm 0.02^{\mathrm{a}}$ \\
\hline & & $\mathrm{C}$ & $3.68 \pm 0.13^{\mathrm{g}}$ & $4.98 \pm 0.02^{\mathrm{a}}$ & $4.60 \pm 0.11^{\mathrm{bc}}$ & $4.98 \pm 0.02^{\mathrm{a}}$ \\
\hline & & $\mathrm{Cr}$ & $4.32 \pm 0.07^{\mathrm{ed}}$ & $4.88 \pm 0.08^{\mathrm{ba}}$ & $4.36 \pm 0.07^{\mathrm{de}}$ & $4.46 \pm 0.09^{\mathrm{e}}$ \\
\hline \multirow{9}{*}{ Oven drying } & \multirow{3}{*}{ Control } & $\mathrm{T}$ & $4.56 \pm 0.07^{\mathrm{dc}}$ & $4.82 \pm 0.05^{\text {bac }}$ & $4.64 \pm 0.07^{\mathrm{bc}}$ & $4.56 \pm 0.07^{\mathrm{de}}$ \\
\hline & & $\mathrm{C}$ & $4.82 \pm 0.05^{\mathrm{ba}}$ & $4.54 \pm 0.10^{\text {ed }}$ & $4.72 \pm 0.06^{\mathrm{bc}}$ & $4.80 \pm 0.06^{\mathrm{bc}}$ \\
\hline & & $\mathrm{Cr}$ & $4.74 \pm 0.07^{\mathrm{bac}}$ & $4.48 \pm 0.07^{\text {ed }}$ & $4.52 \pm 0.07^{\mathrm{dc}}$ & $4.58 \pm 0.07^{\mathrm{de}}$ \\
\hline & \multirow{3}{*}{ Garlic } & $\mathrm{T}$ & $4.18 \pm 0.09^{\mathrm{ef}}$ & $4.90 \pm 0.04^{\mathrm{ba}}$ & $4.52 \pm 0.09^{\mathrm{dc}}$ & $4.72 \pm 0.06^{\mathrm{dc}}$ \\
\hline & & $\mathrm{C}$ & $4.80 \pm 0.06^{\mathrm{bac}}$ & $4.44 \pm 0.10^{\mathrm{e}}$ & $4.94 \pm 0.04^{\mathrm{a}}$ & $4.90 \pm 0.04^{\text {ba }}$ \\
\hline & & $\mathrm{Cr}$ & $4.88 \pm 0.06^{\mathrm{ba}}$ & $4.94 \pm 0.04^{\mathrm{a}}$ & $4.56 \pm 0.08^{\mathrm{dc}}$ & $4.90 \pm 0.04^{\mathrm{ba}}$ \\
\hline & \multirow{3}{*}{ Ginger } & $\mathrm{T}$ & $4.06 \pm 0.08^{f}$ & $4.78 \pm 0.06^{\mathrm{bac}}$ & $4.80 \pm 0.06^{\mathrm{ba}}$ & $4.70 \pm 0.07^{\mathrm{dc}}$ \\
\hline & & $\mathrm{C}$ & $4.72 \pm 0.06^{\mathrm{bc}}$ & $4.62 \pm 0.07^{\mathrm{edc}}$ & $4.70 \pm 0.07^{\mathrm{bc}}$ & $4.80 \pm 0.06^{\mathrm{bc}}$ \\
\hline & & $\mathrm{Cr}$ & $4.90 \pm 0.04^{\mathrm{ba}}$ & $4.70 \pm 0.09^{\mathrm{bdc}}$ & $4.68 \pm 0.11^{\mathrm{bc}}$ & $4.98 \pm 0.02^{\mathrm{a}}$ \\
\hline $\mathrm{CV}$ & & & 14.56 & 12.35 & 12.07 & 9.33 \\
\hline LSD & & & 0.24 & 0.22 & 0.21 & 0.17 \\
\hline
\end{tabular}

Where, $\mathrm{LSD}=$ list significant difference, $\mathrm{CV}=$ coefficient of variation, $\mathrm{Spp} .=$ species, $\mathrm{T}=$ tilapia, $\mathrm{C}=\mathrm{cat}$ fish, $\mathrm{Cr}=$ common carp and the values are mean $\pm \mathrm{SE}$ in that the mean values followed by the same letter in a column are not significantly different at $5 \%$ level of significance. 
Table 17 The effect of storage time on shelf-life of dried fillets

\begin{tabular}{l|lccccc}
\hline \multirow{2}{*}{ Storage Time (Month) } & \multirow{2}{*}{ MD } & \multicolumn{5}{c}{ Nutritional components } \\
\cline { 2 - 6 } & & Ash $(\%, \mathrm{db})$ & Protein $(\%, \mathrm{db})$ & Fat $(\%, \mathrm{db})$ & CHO $(\%, \mathrm{db})$ & GE $(\mathrm{Kcal} / 100 \mathrm{~g})$ \\
\hline \multirow{2}{*}{1} & $\mathrm{~S}$ & $7.31 \pm 0.55^{\mathrm{a}}$ & $75.10 \pm 0.65^{\mathrm{a}}$ & $9.71 \pm 0.19^{\mathrm{a}}$ & $7.88 \pm 0.56^{\mathrm{b}}$ & $419.33 \pm 2.61^{\mathrm{a}}$ \\
& $\mathrm{O}$ & $7.16 \pm 0.29^{\mathrm{a}}$ & $75.65 \pm 0.45^{\mathrm{a}}$ & $7.28 \pm 0.51^{\mathrm{b}}$ & $9.91 \pm 0.68^{\mathrm{a}}$ & $407.76 \pm 1.39^{\mathrm{b}}$ \\
2 & $\mathrm{~S}$ & $7.20 \pm 0.50^{\mathrm{a}}$ & $75.27 \pm 0.69^{\mathrm{a}}$ & $9.69 \pm 0.18^{\mathrm{a}}$ & $7.84 \pm 0.56^{\mathrm{b}}$ & $419.66 \pm 2.37^{\mathrm{a}}$ \\
& $\mathrm{O}$ & $7.05 \pm 0.32^{\mathrm{a}}$ & $75.82 \pm 0.51^{\mathrm{a}}$ & $7.24 \pm 0.50^{\mathrm{b}}$ & $9.89 \pm 0.67^{\mathrm{a}}$ & $408.00 \pm 1.31^{\mathrm{b}}$ \\
3 & $\mathrm{~S}$ & $7.16 \pm 0.47^{\mathrm{a}}$ & $75.37 \pm 0.73^{\mathrm{a}}$ & $9.69 \pm 0.17^{\mathrm{a}}$ & $7.78 \pm 0.61^{\mathrm{b}}$ & $419.81 \pm 2.19^{\mathrm{a}}$ \\
& $\mathrm{O}$ & $7.01 \pm 0.34^{\mathrm{a}}$ & $75.92 \pm 0.59^{\mathrm{a}}$ & $7.23 \pm 0.50^{\mathrm{b}}$ & $9.84 \pm 0.69^{\mathrm{a}}$ & $408.10 \pm 1.36^{\mathrm{b}}$ \\
& $\mathrm{S}$ & $7.17 \pm 0.49^{\mathrm{a}}$ & $75.39 \pm 0.74^{\mathrm{a}}$ & $9.66 \pm 0.18^{\mathrm{a}}$ & $7.78 \pm 0.62^{\mathrm{b}}$ & $419.62 \pm 2.50^{\mathrm{a}}$ \\
& $\mathrm{O}$ & $7.02 \pm 0.35^{\mathrm{a}}$ & $75.94 \pm 0.62^{\mathrm{a}}$ & $7.18 \pm 0.47^{\mathrm{b}}$ & $9.86 \pm 0.68^{\mathrm{a}}$ & $407.82 \pm 1.08^{\mathrm{b}}$ \\
& $\mathrm{CV}$ & 10.24 & 1.44 & 7.60 & 12.44 & 0.81 \\
& LSD & 1.27 & 1.89 & 1.11 & 1.91 & 5.82 \\
\hline
\end{tabular}

Where, $\mathrm{MD}=$ method of drying, $\mathrm{S}=$ sun drying, $\mathrm{O}=$ oven drying, $\mathrm{CHO}=$ carbohydrate, $\mathrm{GE}=$ gross energy, values are mean $\pm \mathrm{SE}$ and mean values followed by the same letter in a column are not significantly different at $5 \%$ level of significance.

The ash contents of the stored fillets were slightly decreased in increasing storage periods from zero months to three months with showing no statistical $(\mathrm{P}<0.05)$ difference in between the storage periods in both methods of drying. Initial and final values of ash contents of stored fillets in sun and oven dried were 7.31 and 7.16 and 7.17 and $7.02 \%$ respectively.

The result obtained by Abdul et al (2011) is supportive to this study showing significant reduction in ash contents as storage period increased. This was also true in findings reported by Magawata and Shina 2013 on garlic and ginger treatments on fish shelf-life. The total carbohydrate in both methods of drying fluctuating from zero month storage up the third month storage. According the Abdul et al (2011), the total carbohydrate showed reduction through the storage of three months. However, the changes of the values of carbohydrate in this study may be due to the increasing and decreasing other components.

\section{Conclusion}

This study was conducted to assess the effect of drying methods and pre-treatments on nutritional value and sensory quality of dried fish. The experiment was conducted in a factorial arrangement of $2 \times 3 \times 2$ with two drying methods (sun and oven drying,) three fish species (tilapia, cat fish and carp) and two preservatives treatment (garlic and ginger juice) laid out in Completely Randomized Design (CRD). Fresh fillets were analyzed for their nutritional value and sensory quality. Fresh fillet and untreated dry fillet were used as control.

The compositions of the fresh fillets were 6.50-7.59\% for ash, $74.20-76.67 \%$ for protein, $8.06-9.09 \%$ for fat and $8.47-9.12 \%$ for carbohydrate. Drying reduced the moisture contents from $74.74-75.81 \%$ to between 7.76$8.25 \%$, making it very safe for long term storage. The ash content changed from 7.11 to 7.34 and from $6.50 \%$ to $6.34 \%$ for cat fish and tilapia, respectively, with statistical significance whereas no change was observed in carp with $7.60 \%$. No difference had been caused in the rest of the compositions due to drying. Drying method had no difference in ash and protein contents while increase in fat from 7.75 to $9.44 \%$ and a decrease in carbohydrate from 9.37 to $8.13 \%$ were observed in sun dried samples than that of oven dried fillets. No difference in composition has been detected in all samples associated with pretreatments except reduction in moisture as compared to the untreated ones.

The present study was conducted to examine the effect of drying methods on nutritional composition, shelf-life and sensory quality of three fish species commonly used in Gambella Regional state, Abobo District. Traditional fish processing around Alwero reservoir in Abobo District produce cheap protein sources for poor population.

\section{References}

Abdul M, Siddique M, Aktar M. 2011. Changes of nutritional value of three marine dry fish (Johnius dussumieri, Harpodon nehereus and Lepturacanthus savala) during Storage.

Akinneye JO, Amoo IA, Arannilewa ST. 2007. Effect of drying methods on nutritional composition of three species of (Bonga sp., Sardinella sp. and Heterotis niloticus) fish. Int., 2(1): 99103.

Ames G, Clucas IJ, Paul SS. 1991. Post harvest losses of fish in the tropics. Report on the natural resources institute $\mathrm{p} 22$.

AOAC. 2000. Official Methods of Analysis of the Association of Official Analytical Chemists (14th Ed.), Washington, p.1193.

Bolaji BO. 2005. Performance evaluation of a simple solar dryer for food preservation. Book of Proceedings of 6th Annual Engineering Technology, p. Federal University of Technology, Minna, Nigeria, pp: 8-13.

Delgade CL, Wada, MWN, Rosegran SM, Ahmed M. 2003. Fish for 2020. Supply and demand in changing global markets. International Food Policy Reseach Institute, Washington, D.C. and World Fish Centre, Penanng, Malaysia.

Eyo AA. 1997. Post harvest losses in the fisheries of Kainji Lake. A consultancy report submitted to Nigerian/German (GTZ) Kainji lake fisheries promotion project, March, pp: 75 .

Eyo, AA. 1986. Significance of fish handling preservation and processing in the development of Nigeria inland fisheries with special reference to Kanji Lake. Fisheries society Nigeria: 3rd annual conference proceedings.

Magawata I, Shina AA. 2013. Effect of spice treatment on the quality of solar dried, Africa cat fish Clarias Gariepinus (Burchell, 1822) In Sokoto, Nigeria.

Mdegela R, Ruttaisire J, Obua J, Okoth S. 2010. Fishery and Aquaculture cluster proceeding workshop.

MoA. 2012. The report done by MOA on the total fish production and productivity.

Okorely and Kwarten, 2006 and Bolaji, 2005. The traditional fish processing activities.

Oladipo IC, Bankole SO. 2013. Nutritional and microbial quality of fresh and dried Clarias gariepinus and Oreochromis niloticus.

Opara LU, Al-Jufiaili SM. 2006. Status of fisheries pot harvest industry in the Sultane of Oman: Part 2 quantification of fresh fish losses. J. Fish. Int., 1(2-4): 150-156.

UNCEF. 2005. Riverside fishing activities in Ethiopia. 